\title{
Asymptotics and Zeros of Sobolev Orthogonal Polynomials on Unbounded Supports
}

\author{
Francisco Marcellán • Juan José Moreno Balcázar
}

\begin{abstract}
In this paper we present a survey about analytic properties of polynomials orthogonal with respect to a weighted Sobolev inner product such that the vector of measures has an unbounded support. In particular, we focus on the asymptotic behaviour of such polynomials as well as in the distribution of their zeros. Some open problems as well as some directions for future research are formulated.
\end{abstract}

Key words Sobolev orthogonal polynomials • asymptotics • zeros.

Mathematics Subject Classifications (2000) Primary 42C05 • Secondary 33C45.

Research of Francisco Marcellán was partially supported by Dirección General de Investigación (Ministerio de Ciencia y Tecnología) of Spain under grant BFM2003-06335-C03-02 and INTAS Research Network NeCCA INTAS 03-51-6637.

Research of Juan José Moreno Balcázar was partially supported by Ministerio de Educación y Ciencia of Spain under grant MTM2005-08648-C02-01 and Junta de Andalucía (FQM 229 and FQM 481).

F. Marcellán $(\bowtie)$

Departamento de Matemáticas, Universidad Carlos III de Madrid, Leganés, Spain e-mail: pacomarc@ing.uc3m.es

\section{J. J. Moreno Balcázar}

Departamento de Estadística y Matemática Aplicada, Universidad de Almería, Almería, Spain

J. J. Moreno Balcázar

Instituto Carlos I de Física Teórica y Computacional, Universidad de Granada, Granada, Spain 


\section{Introduction}

The study of polynomials orthogonal with respect to a Sobolev inner product

$$
(p, q)=\sum_{k=0}^{N} \int p^{(k)}(x) q^{(k)}(x) \mathrm{d} \mu_{k}(x)
$$

where $\mu=\left(\mu_{0}, \mu_{1}, \ldots, \mu_{N}\right)$ is a vector of positive Borel measures supported on the real line and $p, q \in \mathbb{P}$, where $\mathbb{P}$ denotes the set of polynomials with real coefficients, appears in many areas of Applied Mathematics. In smooth data fitting [22] they constitute the natural basis for the approximating subspaces. In Fourier expansions, the analysis of Gibbs phenomena can be performed in a successful way using such polynomials as the numerical experiments given by Iserles et al. [16] show. Finally, the analysis of spectral methods for boundary value problems based on such polynomials is more competitive than the consideration of standard orthogonal polynomials.

The subject has attracted increased attention in the last 15 years. As a sample of it, the extensive bibliography by Marcellán and Ronveaux [30] shows the different topics of interest for researchers. Most of the contributions therein are related to vector of measures $\mu$ such that their components are classical or are like-classical measures, i.e., Jacobi, Laguerre, and Hermite measures. This is a natural consequence of the well known behaviour of the corresponding sequences of orthogonal polynomials from an analytic point of view. In particular, attention was focused on $N=1$ in order to understand the simplest cases.

When an inner product like (1) is considered, two basic properties of standard orthogonal polynomials $(N=0)$ are lost. First, the polynomials orthogonal with respect to (1) with $N>0$ do not satisfy a three-term recurrence relation that constitutes a useful tool in the analysis of the ratio asymptotics of standard orthogonal polynomials. Second, the zeros of polynomials orthogonal with respect to (1) with $N>0$ are not, in general, real and simple as in the standard case. They can be complex and even, if real, they can be located outside the convex hull of the support of the vector of measures $\mu$.

When $\mu$ has bounded support, the asymptotic behaviour of the polynomials orthogonal with respect to (1) has been extensively studied by A. MartínezFinkelshtein, G. López-Lagomasino among others. In particular, relative asymptotics, $n$th root asymptotics, and strong asymptotics have been deeply analyzed.

Nevertheless, when $\mu$ is a vector of measures with unbounded support, despite the examples related to Laguerre weights (the so called coherent pairs) or Hermite weights (the so called symmetrically coherent pairs) very few examples are known. In [10] the case of the vector of measures $\mathrm{d} \mu=\left(e^{-x^{4}} \mathrm{~d} x, \lambda e^{-x^{4}} \mathrm{~d} x\right)$ is analyzed.In particular, relative asymptotics of this Sobolev orthogonal polynomials in terms of the Freud polynomials associated with the weight function $w(x)=\exp \left(-x^{4}\right)$ are deduced. In a more general context, in [18] different kinds of asymptotic behaviour of Sobolev orthogonal polynomials for exponential weights are analyzed in the case $N=1$. In particular, outer and inner asymptotics in terms of $L_{2}$ and $L_{\infty}$ norms as well as strong asymptotics are considered. The key role is played in these problems by the polynomials orthogonal with respect to the measure $\mu_{1}$.

The aim of our paper is to present the state of the art in the area of analytic properties of Sobolev polynomials orthogonal with respect to vector of measures 
of exponential type supported on unbounded sets of the real line. We will focus our attention in the asymptotic behaviour of such polynomials as well as in the distribution of their zeros, emphasizing the comparison with the same properties for standard orthogonal polynomials with respect to the measures $\mu_{0}$ and $\mu_{1}$. Thus we give a survey following the approach of Marcellán et al. [26], Meijer [34] and Martínez-Finkelshtein [31, 32].

We hope it will be useful for people interested in this field not only from a theoretical point of view but taking into account the potential applications.

The structure of the paper is the following. In Section 2, we deal with Sobolev inner products (1) for $N=1$ when one of the measures is Laguerre (resp. Hermite) and the other one is its coherent (resp. symmetrically coherent) companion in the sense of Meijer classification [35]. In the Laguerre case we also consider a nondiagonal inner product connected with the framework of the coherence. In both cases, relative asymptotics in terms of the corresponding classical polynomials as well as strong asymptotics on compact subsets of $(0, \infty)$ (resp. real line) in Laguerre case (resp. in Hermite case) are studied. The distribution of the zeros of such polynomials as well as the location in terms of the zeros of the corresponding classical orthogonal polynomials as well as interlacing and separation properties are deduced. Finally, the behaviour of the zeros of these Sobolev orthogonal polynomials in terms of the zeros of Bessel functions is deduced by means of generalized Mehler-Heine type formulas for Laguerre and Hermite polynomials. Furthermore, in Proposition 2.17 we establish that the natural family of polynomials to compare with these Sobolev polynomials is the family of polynomials defined as $R_{n}(x)=\lim _{\lambda \rightarrow \infty} Q_{n}(x)$. This proves the important role of the parameter $\lambda$.

In Section 3, we leave the framework of coherence. Here, asymptotics properties for polynomials orthogonal with respect to a vector of measures with $\mathrm{d} \mu_{0}=$ $\psi(x) \exp (-Q(x)) \mathrm{d} x, \mathrm{~d} \mu_{1}=\lambda \exp (-Q(x)) \mathrm{d} x$ where $Q$ is an even and continuous function on the real line satisfying some extra conditions and $\psi$ is a measurable and positive function on a set of positive measure on $\mathbb{R}$ are studied. Again the parameter $\lambda$ plays an important role in the asymptotic behaviour of these Sobolev orthogonal polynomials. This is one of the main differences with the case of measures of bounded support.

Finally, in Section 4 some open problems are posed as well as some new directions for a further research in the subject are presented.

\section{The 'Classical' Cases}

We consider the Sobolev inner product

$$
(p, q)=\int p(x) q(x) \mathrm{d} \mu_{0}(x)+\lambda \int p^{\prime}(x) q^{\prime}(x) \mathrm{d} \mu_{1}(x)
$$

where $\mu_{i}(x)$ are measures supported on subsets $I_{i} \subseteq \mathbb{R}$.

We speak of the 'classical' cases when one of the measures involved in the inner product (2) is a classical measure with unbounded support, i.e., the measures corresponding to either the Laguerre or Hermite weight functions. Indeed, this situation is the only one studied for measures with unbounded supports until the work [10] for an example of Freud weights. Three contributions concerning Laguerre and Hermite weights were given in the 1990s (see [27, 37, 41]). These papers are 
in the framework of the concept of coherent and symmetrically coherent pairs of measures introduced in [17] being one of the measures the corresponding to the Laguerre weight. In fact, in 1997 Meijer established the complete classification both of the coherent and symmetrically coherent pairs [35]. In the case of measures with unbounded support we get:

Laguerre weights:

Case I:

- I.1.

$$
\mathrm{d} \mu_{0}=(x+a) x^{\alpha-1} e^{-x} \mathrm{~d} x, \mathrm{~d} \mu_{1}=x^{\alpha} e^{-x} \mathrm{~d} x, \quad a \geq 0, \alpha>0 .
$$

- I.2.

$$
\mathrm{d} \mu_{0}=e^{-x} \mathrm{~d} x+M \delta(x), \mathrm{d} \mu_{1}=e^{-x} \mathrm{~d} x, \quad M \geq 0 .
$$

Case II:

$$
\mathrm{d} \mu_{0}=x^{\alpha} e^{-x} \mathrm{~d} x, \mathrm{~d} \mu_{1}=\frac{x^{\alpha+1} e^{-x}}{x+a} \mathrm{~d} x+M \delta(x+a), \quad a \geq 0, \alpha>-1, \quad M \geq 0 .
$$

Hermite weights:

Case I:

$$
\mathrm{d} \mu_{0}=\left(x^{2}+a^{2}\right) e^{-x^{2}} \mathrm{~d} x, \mathrm{~d} \mu_{1}=e^{-x^{2}} \mathrm{~d} x, \quad a \in \mathbb{R}
$$

Case II:

$$
\mathrm{d} \mu_{0}=e^{-x^{2}} \mathrm{~d} x, \mathrm{~d} \mu_{1}=\frac{e^{-x^{2}}}{x^{2}+a^{2}} \mathrm{~d} x, \quad a \in \mathbb{R} \backslash\{0\} .
$$

Notice that the cases II are rational modifications of the classical measure while the cases I.1 and I for the Laguerre and Hermite weights, respectively, are polynomial modifications of these measures. Therefore, it is natural to think that we will have more problems to establish analytic properties for the Sobolev orthogonal polynomials of Type II because we will need to study families of standard orthogonal polynomials with respect to a rational modification of a measure.

\subsection{Laguerre Weights}

The paper [27] is a very particular case when both measures correspond to the Laguerre weight function. The authors obtain the asymptotic behaviour of these Sobolev orthogonal polynomials uniformly on compact subsets of $\mathbb{C} \backslash[0, \infty)$. In the paper [41] the $n$ th-root asymptotic of the Laguerre-Sobolev polynomials is obtained in $\mathbb{C} \backslash[0,2]$ (although along the paper the sentence "locally uniformly on compact subsets of [0,2]" appears as a misprint). Later on, in [37] the authors deal with the analytic properties of Laguerre-Sobolev orthogonal polynomials of both types. They obtain the asymptotics in $\mathbb{C} \backslash[0, \infty)$. We will introduce these results in the following items. 


\subsubsection{Non-Diagonal Laguerre-Sobolev Orthogonal Polynomials and their Connection with Laguerre-Sobolev of Case I}

In [29] the inner product

$$
(p, q)_{S}=\int_{0}^{\infty}\left(p, p^{\prime}\right)\left(\begin{array}{ll}
1 & \delta \\
\delta & \lambda
\end{array}\right)\left(\begin{array}{l}
q \\
q^{\prime}
\end{array}\right) x^{\alpha} e^{-x} \mathrm{~d} x,
$$

with $\alpha>-1$ is considered. There, the assumption $\lambda, \delta \in \mathbb{R}$ with $\lambda-\delta^{2}>0$ yields the existence of a unique sequence $\left\{Q_{n}^{(N D)}\right\}$ of polynomials orthogonal with respect to (8) up to normalization. In [29] the polynomials $Q_{n}^{(N D)}$ were considered as monic. Here, we denote by $L_{n}^{(\alpha)}(x)=\left((-1)^{n} / n !\right) x^{n}+\ldots$ the classical Laguerre polynomials and we define the polynomials $Q_{n}^{(N D)}$ in such a way they have the same leading coefficient.

This inner product and the corresponding Sobolev orthogonal polynomials are related to Laguerre-Sobolev polynomials of case I.1 whose measures are given by (3) through a straightforward integration by parts. Indeed, for $\alpha>0$, we have

$$
(p, q)_{S}=\int_{0}^{\infty} p(x) q(x) s(x) x^{\alpha-1} e^{-x} \mathrm{~d} x+\lambda \int_{0}^{\infty} p^{\prime}(x) q^{\prime}(x) x^{\alpha} e^{-x} \mathrm{~d} x,
$$

where $s(x)=(1+\delta) x-\alpha \delta$. Therefore, the inner product associated with the measures in (3) is a particular case of (8), i.e., for $\alpha>0$ and $\delta \in(-1,0]$ the inner product

$$
\begin{aligned}
(p, q)_{S *}:= & \frac{1}{(1+\delta)}(p, q)_{S}=\int_{0}^{\infty} p(x) q(x)\left(x-\frac{\alpha \delta}{1+\delta}\right) x^{\alpha-1} e^{-x} \mathrm{~d} x \\
& +\frac{\lambda}{1+\delta} \int_{0}^{\infty} p^{\prime}(x) q^{\prime}(x) x^{\alpha} e^{-x} \mathrm{~d} x:=\int_{0}^{\infty} p(x) q(x)\left(x+a_{1}\right) x^{\alpha-1} e^{-x} \mathrm{~d} x \\
& +\lambda_{1} \int_{0}^{\infty} p^{\prime}(x) q^{\prime}(x) x^{\alpha} e^{-x} \mathrm{~d} x,
\end{aligned}
$$

where $a_{1}, \lambda_{1} \in \mathbb{R}^{+}$, corresponds to the case I.1.

The asymptotic behaviour of these polynomials $Q_{n}^{(N D)}(x)$ and their scaled $Q_{n}^{(N D)}(n x)$ is obtained uniformly on compact subsets of $\mathbb{C} \backslash[0, \infty)$ and $\mathbb{C} \backslash[0,4]$, respectively, i.e.,

Theorem 2.1 (Th. 2 and 4, [29])

(a) Uniformly on compact subsets of $\mathbb{C} \backslash[0, \infty)$,

$$
\lim _{n \rightarrow \infty} \frac{Q_{n}^{(N D)}(x)}{L_{n}^{(\alpha-1)}(x)}=\frac{2(1+\delta)}{\sqrt{\lambda^{2}+4 \lambda(1+\delta)}-\lambda}=\frac{1}{1-(1+\delta) \ell} .
$$

(b) For $\alpha>0$, uniformly on compact subsets of $\mathbb{C} \backslash[0,4]$

$$
\lim _{n \rightarrow \infty} \frac{Q_{n}^{(N D)}(n x)}{L_{n}^{(\alpha-1)}(n x)}=\frac{\varphi\left(\frac{x-2}{2}\right)}{\varphi\left(\frac{x-2}{2}\right)+\ell(1+\delta)}
$$


Furthermore, for $\alpha>-1$,

$$
\lim _{n \rightarrow \infty} \frac{Q_{n}^{(N D)}(n x)}{L_{n}^{(\alpha)}(n x)}=\frac{\varphi\left(\frac{x-2}{2}\right)+1}{\varphi\left(\frac{x-2}{2}\right)+\ell(1+\delta)},
$$

where

$$
\varphi(x)=x+\sqrt{x^{2}-1}
$$

with $\sqrt{x^{2}-1}>0$ if $x>1$, and $\ell$ is given $b y$

$$
\ell=\frac{\lambda+2(1+\delta)-\sqrt{\lambda^{2}+4 \lambda(1+\delta)}}{2(1+\delta)^{2}} \in(0,1) .
$$

\section{Remarks}

- Notice that (10) was obtained in [37, Th.3.5] for the case I.1 if we consider the inner product (9). Furthermore, there (10) is also established for the case I.2 given in (4).

- What are the keys to obtain these results? Indeed, two properties are essential: one is algebraic and the other one is analytic.

1. We have the following relation between the standard polynomials and Sobolev polynomials (see [29, Lemma 1])

$$
L_{n+1}^{(\alpha-1)}(x)=Q_{n+1}^{(N D)}(x)+a_{n} Q_{n}^{(N D)}(x), \quad n \geq 0 .
$$

2. The behaviour of the sequence $a_{n}$ is related to the ratio

$$
\frac{\int_{0}^{\infty}\left(L_{n}^{(\alpha)}(x)\right)^{2} x^{\alpha} e^{-x} \mathrm{~d} x}{\left(Q_{n}^{(N D)}, Q_{n}^{(N D)}\right)_{S}}
$$

when $n$ tends to infinity. Using a non-linear recurrence relation for this ratio (see (17) in [29]) and Poincaré's Theorem the asymptotic result was given in [29, Prop.2].

Then, (10) can be deduced. In order to prove (11) and (12) we need to solve the connection problem for the Laguerre-Sobolev orthogonal polynomials in terms of the Laguerre polynomials, i.e., using (15) in a recursive way we get

$$
Q_{n}^{(N D)}(x)=\sum_{j=0}^{n} \gamma_{j}^{(n)} L_{n-j}^{(\alpha-1)}(x), \quad n \geq 0,
$$

and $\gamma_{0}^{(n)}=1$. Since the asymptotic behaviour of the coefficients $\gamma_{j}^{(n)}$ can be obtained from the asymptotic behaviour of the sequence $\left\{a_{n}\right\}$, then (11) and (12) can be deduced using the asymptotics of scaled Laguerre polynomials $L_{n}^{(\alpha-1)}(n x)$, $L_{n}^{(\alpha)}(n x)$, and Lebesgue's dominated convergence theorem. Although case I.2 was not considered in [29], the same techniques used there allow us to deduce (12) for case I.2. 
- In $[29$, Th.4] to get (11) and (12) it is assumed that $\delta \in(-1,0]$. This condition was posed in order to guarantee a dominant for the sequence $Q_{n}^{(N D)}(n x) / L_{n}^{(\alpha-1)}(n x)$ on compact subsets of $\mathbb{C} \backslash[0,4]$. However, this restriction can be removed by adapting Lemma 3.2 in [5] to our circumstances, and then we get a dominant term for $Q_{n}^{(N D)}(n x) / L_{n}^{(\alpha-1)}(n x)$ for any value of $\delta$.

- From this theorem we can obtain the outer strong asymptotic of the LaguerreSobolev orthogonal polynomials (taking into account the Perron's Theorem) and scaled ones (from a result by Geronimo and Van Assche [19, formula (4.2)]).

In [29] the asymptotics of the sequence $Q_{n}^{(N D)}$ on the oscillatory region is obtained:

Theorem 2.2 (Th. 3, [29]) Uniformly on compact subsets of $(0, \infty)$,

$$
\frac{Q_{n}^{(N D)}(x)}{n^{(\alpha-1) / 2}}=e^{x / 2} x^{-(\alpha-1) / 2} J_{\alpha-1}(2 \sqrt{n x})+O\left(n^{-1 / 4}\right),
$$

where $J_{\alpha-1}(x)$ is the Bessel function

$$
J_{\alpha-1}(x)=\sum_{j=0}^{\infty} \frac{(-1)^{j}(x / 2)^{2 j+\alpha-1}}{j ! \Gamma(j+\alpha)} .
$$

\section{Remarks}

1. To prove the above Theorem the Fejér's formula for Laguerre polynomials (see [42, Th.8.22.1, p.198]) is needed as well as an asymptotic result for the Bessel function $J_{\alpha-1}(x)$, together with the asymptotics of the sequence $a_{n}$.

2. From Theorems 2.1 and 2.2 we can deduce that the non-diagonal LaguerreSobolev polynomials $Q_{n}^{(N D)}$ and, therefore the Laguerre-Sobolev polynomials of case I.1, are asymptotically equivalent to the Laguerre ones of parameter $\alpha-1$.

\subsubsection{Laguerre-Sobolev Orthogonal Polynomials of Case II}

Now we introduce the asymptotic behaviour of the polynomials $Q_{n}^{(L 2)}(x)=$ $\left((-1)^{n} / n !\right) x^{n}+\ldots$ orthogonal with respect to a Sobolev inner product involving the measures given in (5), i.e.,

$$
(p, q)=\int_{0}^{\infty} p(x) q(x) x^{\alpha} e^{-x} \mathrm{~d} x+\lambda \int_{0}^{\infty} p^{\prime}(x) q^{\prime}(x) \frac{x^{\alpha+1} e^{-x}}{x+a} \mathrm{~d} x+M p^{\prime}(-a) q^{\prime}(-a),
$$

where $a \geq 0, \alpha>-1$, and $M \geq 0$.

The first result appears in [37]:

Theorem 2.3 (Th. 4.11, [37]) Relative outer asymptotics.

$$
\lim _{n \rightarrow \infty} \frac{Q_{n}^{(L 2)}(x)}{L_{n}^{(\alpha-1)}(x)}=\left\{\begin{array}{lll}
\frac{1}{1-\ell}\left(1+\frac{a^{1 / 2}}{(-x)^{1 / 2}}\right) & \text { if } & M=0, \\
\frac{1}{1-\ell}\left(1-\frac{a^{1 / 2}}{(-x)^{1 / 2}}\right) & \text { if } & M>0,
\end{array}\right.
$$


uniformly on compact subsets of $\mathbb{C} \backslash[0, \infty)$, where

$$
\ell=\frac{\lambda+2-\sqrt{\lambda^{2}+4 \lambda}}{2} \in(0,1) \text {. }
$$

Remark Notice that (14) is (17) when we consider the inner product (8) as diagonal, that is, $\delta=0$.

In [5] it was proved that the Sobolev polynomials of case II have the same outer scaled asymptotic and the same asymptotics on the oscillatory region as in the case I. It is convenient to remember the expression of the inner product for LaguerreSobolev of type I in terms of non-diagonal Laguerre-Sobolev given by (9).

Theorem 2.4 (Th. 3.1 and 3.3(b) in [5]) Theorem 2.2 and (12) in Theorem 2.1 hold for the polynomials $Q_{n}^{(L 2)}(x)$.

\section{Remarks}

- The key idea of the proofs of Theorems 2.3, 2.4 is the same as in the proof of Theorem 2.1 but more complicated from a technical point of view. Now, we have an algebraic relation:

$$
L_{n}^{(\alpha)}(x)-\sigma_{n-1} L_{n-1}^{(\alpha)}(x)=Q_{n}^{(L 2)}(x)-a_{n-1}^{(L 2)} Q_{n-1}^{(L 2)}(x)
$$

and the analysis of the asymptotic behaviour of the sequence $\sigma_{n}$ is not trivial (see [37]). Furthermore, as it was shown in [5], it is necessary to study the analytic properties of the polynomials $V_{n}(x):=L_{n}^{(\alpha)}(x)-\sigma_{n-1} L_{n-1}^{(\alpha)}(x)$ and one gets $V_{n}^{\prime}(x)=-T_{n-1}(x)$ where $T_{n}(x)=\left((-1)^{n} / n !\right) x^{n}+\ldots$ are the polynomials orthogonal with respect to the standard inner product:

$$
(p, q)=\int_{0}^{\infty} p(x) q(x) \frac{x^{\alpha+1} e^{-x}}{x+a} \mathrm{~d} x+M p(-a) q(-a) .
$$

This family $\left\{T_{n}\right\}$ is orthogonal with respect to a measure whose absolutely continuous part is a rational modification of the Laguerre weight function $x^{\alpha+1} e^{-x}$ on $[0, \infty)$ and possibly with a mass point (a Dirac delta) at $-a \leq 0$.

- One important question arises: why (16) does not yield a constant as in case I? We will come back to this question later on.

\subsubsection{Zeros and its Asymptotics}

In [36] a complete study of the zeros of coherent pairs of Jacobi and Laguerre types using Gaussian quadrature formulas was done. Before stating the results it is important to point out that the notation used in [36] for Laguerre-Sobolev of case I is:

$$
\mathrm{d} \mu_{0}=(x+a) x^{\alpha} e^{-x} \mathrm{~d} x, \mathrm{~d} \mu_{1}=x^{\alpha+1} e^{-x} \mathrm{~d} x, \quad a \geq 0, \alpha>-1,
$$

and so, the change $\alpha \rightarrow \alpha-1$ must be made in the results of [36] for case I to agree with the notation (3). 
Theorem 2.5 (Th. 4.1, 4.2, 4.3, 4.4. and 4.5 in [36]) Let $Q_{n}^{(L 1)}$ be the LaguerreSobolev orthogonal polynomials of case I, i.e., orthogonal with respect to Sobolev inner products induced by the measures given by (3) or (4), then for $n \geq 2$,

- $\quad$ All the zeros of $Q_{n}^{(L 1)}$ are real, simple, and positive.

- The zeros of $Q_{n}^{(L 1)}$ interlace with the zeros of $L_{n}^{(\alpha)}$, i.e., denoting by $l_{n, i}^{(\alpha)}$ and $q_{n, i}^{(L 1)}$ the zeros of $L_{n}^{(\alpha)}$ and $Q_{n}^{(L 1)}$, respectively, then we get

$$
q_{n, 1}^{(L 1)}<l_{n, 1}^{(\alpha)}<q_{n, 2}^{(L 1)}<l_{n, 2}^{(\alpha)}<\ldots<q_{n, n}^{(L 1)}<l_{n, n}^{(\alpha)} .
$$

- $\quad$ For case I.1, the zeros of $Q_{n}^{(L 1)}$ interlace with the zeros of $L_{n}^{(\alpha-1)}$, i.e.,

$$
l_{n, 1}^{(\alpha-1)}<q_{n, 1}^{(L 1)}<l_{n, 2}^{(\alpha-1)}<q_{n, 2}^{(L 1)}<\ldots<l_{n, n}^{(\alpha-1)}<q_{n, n}^{(L 1)}
$$

and for case I.2, the zeros of $Q_{n}^{(L 1)}$ interlace with the zeros of $x L_{n-1}^{(1)}$ as in (19).

- $\quad$ The zeros of $Q_{n-1}^{(L 1)}$ separate the zeros of $Q_{n}^{(L 1)}$.

For case II the results are summarized in the following theorem:

Theorem 2.6 (Th. 4.1 in [36]) Let $Q_{n}^{(L 2)}$ be the Laguerre-Sobolev orthogonal polynomials of case II, then for $n \geq 2$,

- $\quad$ All the zeros of $Q_{n}^{(L 2)}$ are real, simple, and at most one of them is in $(-\infty, 0]$.

- $\quad$ The zeros of $Q_{n}^{(L 2)}$ interlace with the zeros of Laguerre $L_{n}^{(\alpha)}$ in the same sense as (18).

Remarks These results give us bounds for the zeros of Laguerre-Sobolev polynomials independently of the parameter $a$ and $\lambda$. On the other hand, it is important to note that the other results appearing in Theorem 2.5 for case I are not true in general for case II.

In order to obtain asymptotic properties of the zeros of Laguerre-Sobolev polynomials we obtain Mehler-Heine type formulas (see [42, p.193]).

The study of the asymptotics of the zeros of Laguerre-Sobolev polynomials was done in the papers [29] for case I.1 and in [5] for case II. The results were obtained from some generalizations of the Mehler-Heine formula for Laguerre polynomials.

Theorem 2.7 (Th. 5 in [29] and Th. 3.3 (a) in [5])

(a) Let $Q_{n}^{(N D)}$ be the polynomials orthogonal with respect to (8) and $\alpha>-1$,

$$
\lim _{n \rightarrow \infty} \frac{Q_{n}^{(N D)}(x / n)}{n^{\alpha-1}}=\frac{1}{1-(1+\delta) \ell} x^{-(\alpha-1) / 2} J_{\alpha-1}(2 \sqrt{x}) .
$$

holds uniformly for $x$ on compact subsets of $\mathbb{C}$ and $\ell$ is given by (14). 
(b) Let $Q_{n}^{(L 2)}$ be the Laguerre-Sobolev orthogonal polynomials of case II. If a $>0$, then

$$
\lim _{n \rightarrow \infty} \frac{Q_{n}^{(L 2)}(x / n)}{n^{\alpha-1 / 2}}=-\frac{\mathrm{d}(a)}{1-\ell} x^{-\alpha / 2} J_{\alpha}(2 \sqrt{x}) .
$$

If $a=0$ and $M>0$, then

$$
\lim _{n \rightarrow \infty} \frac{Q_{n}^{(L 2)}(x / n)}{n^{\alpha-1}}=\frac{1}{1-\ell} s(x) .
$$

Finally, if $a=M=0$, then [20] holds with $\delta=0$.

For $\ell$ given by (17),

$$
\mathrm{d}(a)=\left\{\begin{array}{lll}
-\sqrt{a} & \text { if } & M=0, \\
\sqrt{a} & \text { if } & M>0
\end{array}\right.
$$

and

$$
s(x)=x^{-(\alpha-1) / 2} J_{\alpha-1}(2 \sqrt{x})-(\alpha+1) x^{-\alpha / 2} J_{\alpha}(2 \sqrt{x}) .
$$

All the limits hold uniformly on compact subsets of $\mathbb{C}$.

Remarks As we have explained in the third remark of Theorem 2.1, the restriction on $\delta$ that appears in [29] can be removed. On the other hand, [20] holds for the Laguerre-Sobolev polynomials of case I: it is clear for case I.1 as we already know and it follows in a straightforward way for case I.2.

Now, we can obtain a limit relation between the zeros of Laguerre-Sobolev polynomials and the zeros of Bessel functions using Hurwitz's theorem in the results of this theorem. Thus we get

Corollary 2.8 (Prop. 4 in [29] and Cor. 3.4 in [5])

(a) Let $q_{n, i}^{(N D)}$ be the zeros of $Q_{n}^{(N D)}$. Then

$$
\lim _{n \rightarrow \infty} n q_{n, i}^{(N D)}=\frac{j_{\alpha-1, i}^{2}}{4},
$$

where three cases appear:

- If $-1<\alpha<0, j_{\alpha-1,1}$ is any of the two purely imaginary zeros of $J_{\alpha-1}(x)$ and, for $i \geq 2, j_{\alpha-1, i}$ is the $(i-1)$ th positive real zero of $J_{\alpha-1}(x)$.

- If $\alpha=0, j_{\alpha-1,1}=j_{-1,1}=0$ and, for $i \geq 2, j_{-1, i}$ is the $(i-1)$ th positive real zero of $J_{-1}(x)$.

- If $\alpha>0, j_{\alpha-1, i}$ is the $i$ th positive real zero of $J_{\alpha-1}(x)$.

(b) Let $q_{n, i}^{(L 2)}$ be the zeros of $Q_{n}^{(L 2)}$.

- If $a>0$, then we have

$$
\lim _{n \rightarrow \infty} n q_{n, i}^{(L 2)}=\frac{j_{\alpha, i}^{2}}{4}
$$

where $j_{\alpha, i}$ the $i$ th positive zero of the Bessel function $J_{\alpha}(x)$. 
- If $a=0$ and $M>0$, then we have

$$
\lim _{n \rightarrow \infty} n q_{n, i}^{(L 2)}=s_{\alpha, i}
$$

where $s_{\alpha, i}$ denotes the $i$ th real zero of function $s(x)$ defined in (21).

- If $a=M=0$, then (22) holds.

\section{Remarks}

- Notice that for the zeros of Laguerre-Sobolev polynomials of case I, (22) holds. Indeed, cases I.1 and I.2 correspond to the third and second case, respectively.

- The Laguerre-Sobolev polynomials of case II have exactly one negative zero for $n$ sufficiently large if and only if either $\alpha>-1, a=0$, and $M>0$ or $-1<\alpha<$ 0 and $a=M=0$.

- For a fixed $n$, it would be very interesting to analyze the behaviour of $q_{n, j}^{(L k)}$ $k=1,2$ in terms of the parameter $\lambda$.

- The function $s(x)$ given by (21) has only one negative zero and their positive zeros separate those of $J_{\alpha+1}(2 \sqrt{x})$.

- From this theorem we can get information about the critical points. In fact, the critical points of these families of polynomials for $n$ sufficiently large lie on $[0,+\infty)$.

\subsubsection{A Generalization of Laguerre Polynomials: The Laguerre-Sobolev Type}

In [20] the inner product

$$
(p, q)=\frac{1}{\Gamma(\alpha+1)} \int_{0}^{\infty} p(x) q(x) x^{\alpha} e^{-x} \mathrm{~d} x+M p(0) q(0)+N p^{\prime}(0) q^{\prime}(0),
$$

where $M, N \geq 0$ and $\alpha>-1$ was considered. The polynomials $\left(L_{n}^{(\alpha, M, N)}(x)\right)_{n}$ orthogonal with respect to (23) constitute a natural extension of the so-called Koornwinder's generalized Laguerre polynomials which are orthogonal with respect to (23) where $N=0$. Algebraic and differential properties of the generalized Laguerre polynomials $\left(L_{n}^{(\alpha, M, N)}(x)\right)_{n}$ have been obtained in the 90's of the past century. However, the asymptotic properties of $\left(L_{n}^{(\alpha, M, N)}(x)\right)_{n}$ were not known until its consideration in [6].

The asymptotics of these polynomials is obtained in $\mathbb{C} \backslash[0, \infty)$ as well as in the oscillatory region $(0, \infty)$. In [6], following the notation of [20], $L_{n}^{(\alpha)}(x)$ and $L_{n}^{(\alpha, M, N)}(x)$ have as leading coefficients, respectively, $(-1)^{n} / n$ ! and

$$
\frac{(-1)^{n}}{n !}\left(B_{0}(n)-n B_{1}(n)+n(n-1) B_{2}(n)\right),
$$

where

$$
\begin{aligned}
& B_{0}(n)=1-\frac{N}{\alpha+1}\left(\begin{array}{c}
n+\alpha+1 \\
n-2
\end{array}\right), B_{1}(n)=-\frac{M}{\alpha+1}\left(\begin{array}{c}
n+\alpha \\
n
\end{array}\right)-\frac{(\alpha+2) N}{(\alpha+1)(\alpha+3)}\left(\begin{array}{c}
n+\alpha \\
n-2
\end{array}\right), \\
& B_{2}(n)=\frac{N}{(\alpha+1)(\alpha+2)(\alpha+3)}\left(\begin{array}{c}
n+\alpha \\
n-1
\end{array}\right)+\frac{M N}{(\alpha+1)^{2}(\alpha+2)(\alpha+3)}\left(\begin{array}{c}
n+\alpha \\
n
\end{array}\right)\left(\begin{array}{c}
n+\alpha+1 \\
n-1
\end{array}\right) .
\end{aligned}
$$


But if we take $(-1)^{n} / n$ ! as leading coefficient in both families of polynomials then the computations can be simplified. For instance, in the following theorem we summarize some of the results obtained in [6]:

Theorem 2.9 (Th. 1 and 2(a) in [6])

(a) Relative asymptotics.

$$
\lim _{n \rightarrow \infty} \frac{L_{n}^{(\alpha, M, N)}(x)}{L_{n}^{(\alpha)}(x)}=1, \quad \text { with } \quad M, N \geq 0, \alpha>-1,
$$

uniformly on compact subsets of $\mathbb{C} \backslash[0,+\infty)$.

(b) Strong asymptotics on compact subsets of $(0,+\infty)$.

Denote by

$$
g_{n, i}(x)=x^{-\alpha / 2} J_{\alpha+2 i}(2 \sqrt{n x})
$$

then

$$
\begin{aligned}
& \frac{L_{n}^{(\alpha, M, N)}(x)}{n^{\alpha / 2}}= \\
& \begin{cases}c_{1}(n) e^{x / 2} g_{n-1,1}(x)+O\left(n^{-\min \{\alpha+5 / 4,3 / 4\}}\right), & M>0, N=0 ; \\
c_{2}(n) e^{x / 2}\left(a_{2}(n) g_{n-2,2}(x)-(\alpha+2) a_{1}(n) g_{n-1,1}(x)\right. & \\
\left.-a_{0}(n) g_{n, 0}(x)\right)+O\left(n^{-3 / 4}\right), & M=0, N>0 ; \\
c_{3}(n) e^{x / 2} g_{n-2,2}(x)+O\left(n^{-\min \{\alpha+5 / 4,3 / 4\}}\right), & M, N>0,\end{cases}
\end{aligned}
$$

where $\lim _{n \rightarrow \infty} c_{i}(n)=1, i=1,3, \lim _{n \rightarrow \infty} c_{2}(n)=1 /(\alpha+2)$, and $\lim _{n \rightarrow \infty} a_{i}(n)=$ $1, i=0,1,2$.

(c) Relative outer asymptotics of scaled polynomials.

$$
\lim _{n \rightarrow \infty} \frac{L_{n}^{(\alpha, M, N)}(n x)}{L_{n}^{(\alpha)}(n x)}=1, \quad \text { with } \quad M, N \geq 0, \alpha>-1,
$$

uniformly on compact subsets of $\mathbb{C} \backslash[0,4]$.

Remark It is important to note that we have quite simplified the results appearing in [6] as well as we have corrected some misprints, in particular one of them in the scaled asymptotics for the case $M=0, N>0$. For all of them, now it is more easy to understand the meaning of the results.

Concerning their zeros, in [20] it was proved that the zeros of polynomials $L_{n}^{(\alpha, M, N)}(x)$ are real and at least $n-1$ of them lie in $(0, \infty)$. Furthermore, when $N>0$ and $n$ is large enough there exists exactly one zero in $(-\infty, 0]$. We denote by $q_{n, i}$ the zeros of $L_{n}^{(\alpha, M, N)}(x)$. Now, let $N>0$ and $n$ sufficiently large such that $L_{n}^{(\alpha, M, N)}(x)$ has the zero $q_{n, 1}$ in $(-\infty, 0]$. Then, for $M>0$,

$$
-\frac{1}{2}\left(\frac{N}{M}\right)^{1 / 2} \leq q_{n, 1} \leq 0 .
$$


It would be very interesting to analyze the behaviour of $q_{n, 1}$ in terms of $N$, i.e., $q_{n, 1}=$ $O\left(N^{-\mu}\right)$ with $\mu \in \mathbb{R}^{+}$, as well as, the behaviour of $q_{n, j}$ in terms of $N$, for a fixed $M$. Notice that for $N=0$, a standard case, $q_{n, 1}=O\left(M^{-1}\right)$, and $q_{n, j}$ is a decreasing function in terms of $M$.

Later on in [6] asymptotic properties of the zeros were looked for. So MehlerHeine type formulas were obtained and therefore limit relations between the zeros of generalized Laguerre polynomials and the zeros of Bessel functions were also deduced. We summarize the results as:

Proposition 2.10 (Th. 2 (b) and 3 in [6]) If we denote

$$
g_{i}(x)=x^{-\alpha / 2} J_{\alpha+2 i}(2 \sqrt{x})
$$

then

$$
\lim _{n \rightarrow \infty} \frac{L_{n}^{(\alpha, M, N)}(x / n)}{n^{\alpha}}= \begin{cases}-g_{1}(x), & M>0, N=0 ; \\ \frac{1}{\alpha+2}\left(g_{2}(x)-(\alpha+2) g_{1}(x)-g_{0}(x)\right) & M=0, N>0 ; \\ g_{2}(x), & M, N>0 .\end{cases}
$$

uniformly on compact subsets of $\mathbb{C}$. Therefore, denoting by $j_{\alpha, i}$ the $i$-th positive zero of the Bessel function $J_{\alpha}(x)$ and by $q_{n, i}$ the zeros in increasing order of the polynomial $L_{n}^{(\alpha, M, N)}(x)$ with $\alpha>-1$,

(a) If $M>0$ and $N=0$, then

$$
\lim _{n \rightarrow \infty} n q_{n, 1}=0 \quad \text { and } \quad \lim _{n \rightarrow \infty} n q_{n, i}=\frac{j_{\alpha+2, i-1}^{2}}{4}, i \geq 2 .
$$

(b) If $M=0$ and $N>0$, then

$$
\lim _{n \rightarrow \infty} n q_{n, i}=z_{i}
$$

where $z_{i}$ denotes the $i$-th real zero of the function $g(x)=g_{2}(x)-(\alpha+2) g_{1}(x)-$ $g_{0}(x)$ with $g_{i}(x)$ given by (24). Moreover, $g(x)$ has only one negative real zero.

(c) If $M, N>0$, then

$$
\lim _{n \rightarrow \infty} n q_{n, i}=0, i=1,2 \text { and } \lim _{n \rightarrow \infty} n q_{n, i}=\frac{j_{\alpha+4, i-2}^{2}}{4}, i \geq 3
$$

Remarks Notice that for $M>0, N=0$ the first scaled zero, $n q_{n, 1}$, of the orthogonal polynomials (Koornwinder polynomials) $L_{n}^{(\alpha, M, 0)}(x)$ tends to 0 for $n \rightarrow \infty$. On the other hand, in the case $M>0, N>0$ the two first scaled zeros $n q_{n, i}, i=1,2$, with $q_{n, 1}<0$, also tend to 0 when $n \rightarrow \infty$. In both cases the results agree with those of [1] in a more general framework. However, in the case $M=0, N>0$ for $n$ large enough, the first scaled zero $n q_{n, 1}$ is always negative and does not tend to zero when $n \rightarrow \infty$, i.e., $\lim _{n \rightarrow \infty} n q_{n, 1}=z_{1}$ is a negative real number. 
In [6] a conjecture was stated for the polynomials $L_{n}^{\left(\alpha, M_{0}, \ldots, M_{s}\right)}(x)$ orthogonal with respect to the inner product

$$
(p, q)=\frac{1}{\Gamma(\alpha+1)} \int_{0}^{\infty} p(x) q(x) x^{\alpha} e^{-x} \mathrm{~d} x+\sum_{i=0}^{s} M_{i} p^{(i)}(0) q^{(i)}(0), \quad \alpha>-1 .
$$

Now, after the simplifications made in the results of [6], we can formulate it as follows:

Conjecture. If $M_{i}>0, i=0, \ldots, s$ with $s \geq 2$, then

$$
\lim _{n \rightarrow \infty} \frac{L_{n}^{\left(\alpha, M_{0}, \ldots, M_{s}\right)}(x / n)}{n^{\alpha}}=(-1)^{s+1} g_{s+1}(x)
$$

uniformly on compact subsets of $\mathbb{C}$ where $g_{s+1}(x)$ is given by (24).

From the above proposition we can not formulate this conjecture when some of the constants $M_{i}, i=0, \ldots, s-1$, vanish with $M_{s}>0$. What occurs in this case? This is an interesting unsolved problem.

\subsection{Hermite Weights}

Next we consider the inner product

$$
(p, q)_{S}=\int_{-\infty}^{\infty} p(x) q(x) \mathrm{d} \mu_{0}+\lambda \int_{-\infty}^{\infty} p^{\prime}(x) q^{\prime}(x) \mathrm{d} \mu_{1}
$$

where $\left(\mu_{0}, \mu_{1}\right)$ is a symmetrically coherent pair of measures (see [35]) corresponding to Hermite weight, that is, the measures are given by (6) (case I) or (7) (case II) according to the classification given by Meijer, i.e.,

$$
\begin{aligned}
& \mathrm{d} \mu_{0}=\left(x^{2}+a^{2}\right) e^{-x^{2}} \mathrm{~d} x, \mathrm{~d} \mu_{1}=e^{-x^{2}} \mathrm{~d} x, \quad a \in \mathbb{R}, \quad \text { or } \\
& \mathrm{d} \mu_{0}=e^{-x^{2}} \mathrm{~d} x, \quad \mathrm{~d} \mu_{1}=e^{-x^{2}} /\left(x^{2}+a^{2}\right) \mathrm{d} x, a \in \mathbb{R} \backslash\{0\} .
\end{aligned}
$$

In this section we use the notation: $H_{n}(x)=2^{n} x^{n}+\ldots$ for the classical Hermite polynomials and $Q_{n}^{(H 1)}(x)=2^{n} x^{n}+\ldots, Q_{n}^{(H 2)}(x)=2^{n} x^{n}+\ldots$ for the Sobolev polynomials orthogonal with respect to $(25)$ where the measures are given by $(6,7)$, respectively.

In the framework of symmetrically coherent pairs, we have the algebraic relation between the classical Hermite polynomials and the Sobolev orthogonal polynomials (see, for example, $[17$, Th.4(c)])

$$
H_{n+2}(x)+\sigma_{n+1}^{(H i)} H_{n}(x)=Q_{n+2}^{(H i)}(x)+a_{n}^{(H i)} Q_{n}^{(H i)}(x), \quad n \geq i-1, \quad i=1,2,
$$

where $\sigma_{n}^{(H 1)}=0$ for all $n \in \mathbb{N}$.

As in the Laguerre case the keys to obtain the asymptotic behaviour of the Sobolev orthogonal polynomials are related to the limits of the sequences $\sigma_{n}^{(H 2)}$ and 
$a_{n}^{(H i)}, i=1,2$, when $n \rightarrow \infty$. It is important to note that the sequences $a_{n}^{(H 1)}$ and $a_{n}^{(H 2)}$ involve the ratios

$$
\begin{gathered}
\frac{\int_{-\infty}^{\infty} H_{n}^{2}(x) e^{-x^{2}} \mathrm{~d} x}{\left(Q_{n}^{(H 1)}, Q_{n}^{(H 1)}\right)_{S}}, \\
\frac{\int_{-\infty}^{\infty} W_{n}^{2}(x) \frac{e^{-x^{2}}}{x^{2}+a^{2}} \mathrm{~d} x}{\left(Q_{n}^{(H 2)}, Q_{n}^{(H 2)}\right)_{S}},
\end{gathered}
$$

respectively, where $W_{n}(x)=2^{n} x^{n}+\ldots$ are the polynomials orthogonal with respect to the weight function

$$
\frac{e^{-x^{2}}}{x^{2}+a^{2}}, \quad a \neq 0 .
$$

From a technical point of view the case I is more simple than case II. In fact, in [4, Lemma 2.2, 2.4 and 2.6] we get:

$$
\lim _{n \rightarrow \infty} \frac{\sigma_{n}^{(H 2)}}{2 n}=1, \quad \lim _{n \rightarrow \infty} \frac{a_{n}^{(H i)}}{2 n}=\frac{1}{\varphi(2 \lambda+1)}, \quad i=1,2,
$$

where $\varphi$ is given by (13). This is enough to deduce the relative asymptotics between Sobolev orthogonal polynomials and Hermite polynomials in case I. However, for case II more information about the asymptotics of $\sigma_{n}^{(H 2)} /(2 n)$ is needed to get an useful result. So, in [39, Prop. 2] it was deduced

$$
\lim _{n \rightarrow \infty} \sqrt{[n / 2]}\left(\frac{\sigma_{n}^{(H 2)}}{2 n}-1\right)=-|a| .
$$

From it we get:

Theorem 2.11 (Th. 2.3 in [4] and Th. 2 in [39]) Let

$$
\theta(\lambda)=\frac{\varphi(2 \lambda+1)}{\varphi(2 \lambda+1)-1} .
$$

(a) Outer relative asymptotics.

$$
\lim _{n} \frac{Q_{n}^{(H 1)}(x)}{H_{n}(x)}=\theta(\lambda),
$$

uniformly on compact subsets of $\mathbb{C} \backslash \mathbb{R}$.

(b) Outer relative asymptotics.

$$
\lim _{n \rightarrow \infty} \sqrt{\left[\frac{n}{2}\right]} \frac{Q_{n}^{(H 2)}(x)}{H_{n}(x)}=\left\{\begin{array}{cc}
\theta(\lambda)(-i x+|a|) & \text { if } x \in C_{+}, \\
\theta(\lambda)(i x+|a|) & \text { if } x \in C_{-},
\end{array}\right.
$$

uniformly on compact subsets of half planes $C_{+}:=\{x=\alpha+i \beta \in \mathbb{C}: \beta>0\}$ and $C_{-}:=\{x=\alpha+i \beta \in \mathbb{C}: \beta<0\}$, respectively.

Again, $\varphi$ is given by (13). 
Remark As in the Laguerre case, in the relative asymptotic we get as limit a constant for case I and a function depending on $x$ for case II. Thus, why these different situations appear? Later on, we will come back on this question.

On the other hand, the asymptotic of scaled Hermite polynomials was obtained in $[4$, Th. 3.1]:

Theorem 2.12 (Th. 3.1 in [4])

(a) Outer relative asymptotics for scaled polynomials.

$$
\lim _{n} \frac{Q_{n}^{(H 1)}(\sqrt{n} x)}{H_{n}(\sqrt{n} x)}=\frac{\varphi(2 \lambda+1) \varphi^{2}\left(\frac{x}{\sqrt{2}}\right)}{\varphi(2 \lambda+1) \varphi^{2}\left(\frac{x}{\sqrt{2}}\right)+1}
$$

uniformly on compact subsets of $\mathbb{C} \backslash[-\sqrt{2}, \sqrt{2}]$.

(b) Outer relative asymptotics for scaled polynomials.

$$
\lim _{n} \frac{Q_{n}^{(H 2)}(\sqrt{n} x)}{H_{n}(\sqrt{n} x)}=\frac{\left(\varphi^{2}\left(\frac{x}{\sqrt{2}}\right)+1\right) \varphi(2 \lambda+1)}{\varphi(2 \lambda+1) \varphi^{2}\left(\frac{x}{\sqrt{2}}\right)+1}
$$

uniformly on compact subsets of $\mathbb{C} \backslash[-\sqrt{2}, \sqrt{2}]$.

Again, $\varphi$ is given by (13).

It is important to note that for the scaled asymptotics of case II it is enough to know (27). However, for the non-scaled asymptotics obtained later in [39] some extra information about the sequence $\sigma_{n}^{(H 2)}$ given by (28) is needed. This follows from the fact that

$$
\begin{aligned}
\lim _{n \rightarrow \infty} \frac{H_{n+2}(x)+\sigma_{n+1}^{(H 2)} H_{n}(x)}{H_{n+2}(x)} & =0, \\
\lim _{n \rightarrow \infty} \frac{H_{n+2}(\sqrt{n} x)+\sigma_{n+1}^{(H 2)} H_{n}(\sqrt{n} x)}{H_{n+2}(\sqrt{n} x)} & \neq 0,
\end{aligned}
$$

uniformly on compact subsets of $\mathbb{C} \backslash \mathbb{R}$ and of $\mathbb{C} \backslash[-\sqrt{2}, \sqrt{2}]$, respectively.

Next we give the inner asymptotics of the Sobolev polynomials $S_{n}^{(H i)}(x), i=1,2$.

Theorem 2.13 (Th. 2.3 in [12]) Denote $\lambda_{n}^{*}=\frac{\Gamma(n / 2+1)}{\Gamma(n)}$.

(a) For $n \rightarrow \infty$, the polynomial $Q_{n}^{(H 1)}$ satisfies

$$
\lambda_{n}^{*} e^{-\frac{1}{2} x^{2}} Q_{n}^{(H 1)}(x)=\theta(\lambda) F_{n}(x)+o(1), \quad x \in \mathbb{R},
$$

where $F_{n}(x)=\lambda_{n}^{*} e^{-\frac{1}{2} x^{2}} H_{n}(x)$.

(b) For $n \rightarrow \infty$, the polynomial $Q_{n}^{(H 2)}$ satisfies

$$
\frac{\lambda_{n-1}^{*}}{2} e^{-\frac{1}{2} x^{2}} Q_{n}^{(H 2)}(x)=\theta(\lambda) G_{n}(x)+o(1), \quad x \in \mathbb{R},
$$

where $G_{n}(x)=\left\{\lambda_{n-1}^{*} x H_{n-1}(x)-|a| \lambda_{n-2}^{*} H_{n-2}(x)\right\} e^{-\frac{1}{2} x^{2}}$. 
In both cases the convergence is uniform on compact intervals of the real line and $\theta(\lambda)$ is given by (29).

\section{Remarks}

- Notice that in [42, p.200] the asymptotic behaviour of $F_{n}$ is given, that is, for $n \rightarrow \infty$ we have

$$
F_{n}(x)=\cos \left(\sqrt{2 n+1} x-\frac{n \pi}{2}\right)+\mathcal{O}\left(\frac{1}{\sqrt{n}}\right)
$$

uniformly on compact intervals of the real line. Furthermore, it is to observe that $G_{n}(x)=x F_{n-1}(x)-|a| F_{n-2}(x)$ and so, for $n \rightarrow \infty$, we get

$$
G_{n}(x)=|a| \cos \left(\sqrt{2 n-3} x-\frac{n \pi}{2}\right)-x \sin \left(\sqrt{2 n-1} x-\frac{n \pi}{2}\right)+\mathcal{O}\left(\frac{1}{\sqrt{n}}\right),
$$

uniformly on compact intervals of the real line.

- In [11, Prop. 2] some bounds depending of the degree of the polynomials were obtained for $\left|Q_{n}^{(H i)}(x)\right|, i=1,2$, with $x \in \mathbb{R}$.

- We also want to emphasize the fact that analytical properties of HermiteSobolev polynomials of case II involve the study of these properties for the polynomials $W_{n}$ which are orthogonal with respect to a rational modification of Hermite weight function. So, the study of the zeros of the family of orthogonal polynomials $W_{n}$ is associated with Gaussian quadrature formulas for the measure $e^{-x^{2}} /\left(x^{2}+a^{2}\right) \mathrm{d} x$.

\subsubsection{Zeros and its Asymptotics}

The zeros of Hermite-Sobolev polynomials were studied in [13]. Applying Gaussian quadrature formulas and other technical tools the location of the zeros of HermiteSobolev polynomials for both cases I and II is deduced. We summarize the results as:

Theorem 2.14 (Th. 4.7, 4.8, 4.10, 5.7, 5.8, 5.10 in [13]) Let $h_{n, 1}<\ldots<h_{n, m}$ denote the positive zeros of Hermite polynomials $H_{n}$. Then

- $\quad$ The zeros of $Q_{n}^{(H 1)}$ and $Q_{2 n+1}^{(H 2)}$ are real and simple. $Q_{2 n}^{(H 2)}$ has at least $2 n-2$ real and simple zeros.

- Let $p_{n, 1}<\ldots<p_{n, m}$ denote the positive zeros of polynomials orthogonal with respect to the weight function $\left(x^{2}+a^{2}\right) e^{-x^{2}}$ and let $q_{n, 1}^{(H 1)}<\ldots<q_{n, m}^{(H 1)}$ denote the positive zeros of the polynomials $Q_{n}^{(H 1)}$. Then, for $n \geq 3$,

$$
\begin{gathered}
h_{n, 1}<q_{n, 1}^{(H 1)}<\ldots<h_{n, m}<q_{n, m}^{(H 1)}, \\
q_{n, 1}^{(H 1)}<p_{n, 1}<\ldots<q_{n, m}^{(H 1)}<p_{n, m} .
\end{gathered}
$$


- $\quad$ For $n \geq 3$, the zeros of $Q_{n-1}^{(H 1)}$ separate the zeros of $Q_{n}^{(H 1)}$.

- Let $q_{n, 2}^{(H 2)}<\ldots<q_{n, m}^{(H 2)}$ denote the $m-1$ largest positive zeros of polynomials $Q_{n}^{(H 2)}$. Then,

$$
\begin{aligned}
h_{n, 1}<q_{n, 2}^{(H 2)}<h_{n, 2}<\ldots<h_{n, m-1}<q_{n, m}^{(H 2)}<h_{n, m}, \quad n \geq 3, \\
h_{n-2,1}<q_{n, 2}^{(H 2)}<\ldots<h_{n-2, m-1}<q_{n, m}^{(H 2)}, \quad n \geq 4 .
\end{aligned}
$$

Furthermore, if $Q_{n}^{(H 2)}$ has $n$ real and simple zeros and denoting by $q_{n, 1}^{(H 2)}$ the smallest positive zero, then for $n \geq 3$ we have $q_{n, 1}^{(H 2)}<h_{n, 1}$.

\section{Remarks}

- In [13, Th. 5.15] it is also shown that the zeros of the polynomials $W_{2 n}$ orthogonal with respect to the weight (26) interlace with those of $Q_{2 n+1}^{(H 2)}$ and the zeros of $W_{2 n+1}$ interlace with those real zeros of $Q_{2 n+2}^{(\mathrm{H} 2)}$.

- We can see that this theorem is very similar to Theorems 2.5 and 2.6 for the Laguerre case. In fact, the possibility of one negative zero for Laguerre-Sobolev polynomials of case II is, in some sense, equivalent to the possibility of two conjugate complex zeros for even Hermite-Sobolev polynomials of case II.

- $Q_{2 n}^{(H 2)}$ can have complex zeros. For example if we take $a^{2}=1 / 25$ and $\lambda=1$, then the polynomials $Q_{2 n}^{(H 2)}$ have two complex zeros for $n=3, \ldots, 6$, as the following table shows

\begin{tabular}{ll}
\hline$Q_{2 n}$ & Complex zeros \\
\hline$Q_{6}$ & $\pm 0.1399747870 i$ \\
$Q_{8}$ & $\pm 0.1158504270 i$ \\
$Q_{10}$ & $\pm 0.0769761729 i$ \\
$Q_{12}$ & $\pm 0.0308661279 i$ \\
\hline
\end{tabular}

In [13] a study of the zeros of $Q_{2 n}^{(H 2)}$ has been made when $\lambda \rightarrow \infty$. In this paper the authors state that the limit polynomials: $R_{2 n}^{(H 2, \infty)}(x):=\lim _{\lambda \rightarrow \infty} Q_{2 n}^{(H 2)}(x)$ for $n \geq 2$, have complex zeros when $|a| \sqrt{n-1}<0.21$ and have no complex zeros when $|a| \sqrt{n-1}>2.5$. An open question about the complex zeros of these polynomials is posed in [13]: what happens if $0.21<|a| \sqrt{n-1}<2.5$ ?

- For a fixed $n$, and taking into account (31) and (32) we conjecture that $q_{n, k}^{(H j)}$ is an increasing function of $\lambda$ for each $k$.

The study of the asymptotics of the zeros of Hermite-Sobolev polynomials has been done in the papers [11] for case I and in [39] for case II. As in the Laguerre case, the results have been obtained from some generalizations of the Mehler-Heine type formulas for Hermite polynomials. 
Theorem 2.15 (Th. 1 in [11] and Th. 1 in [39])

(a) Let $Q_{n}^{(H 1)}$ be the Hermite-Sobolev orthogonal polynomials of case I. Then,

$$
\begin{gathered}
\lim _{n \rightarrow \infty} \frac{(-1)^{n} \sqrt{n} Q_{2 n}^{(H 1)}(x /(2 \sqrt{n}))}{2^{2 n} n !}=\theta(\lambda) \frac{\cos (x)}{\sqrt{\pi}}, \\
\lim _{n \rightarrow \infty} \frac{(-1)^{n} Q_{2 n+1}^{(H 1)}(x /(2 \sqrt{n}))}{2^{2 n+1} n !}=\theta(\lambda) \frac{\sin (x)}{\sqrt{\pi}},
\end{gathered}
$$

hold both uniformly on compact subsets of $\mathbb{C}$.

(b) Let $Q_{n}^{(H 2)}$ be the Hermite-Sobolev orthogonal polynomials of case II. Then,

$$
\begin{aligned}
\lim _{n \rightarrow \infty} \frac{(-1)^{n} Q_{2 n}^{(H 2)}(x /(2 \sqrt{n}))}{2^{2 n}(n-1) !} & =\theta(\lambda) \frac{|a| \cos (x)}{\sqrt{\pi}}, \\
\lim _{n \rightarrow \infty} \frac{(-1)^{n} \sqrt{n} Q_{2 n+1}^{(H 2)}(x /(2 \sqrt{n}))}{2^{2 n+1} n !} & =\theta(\lambda) \frac{|a| \sin (x)}{\sqrt{\pi}},
\end{aligned}
$$

hold both uniformly on compact subsets of $\mathbb{C}$.

Again, $\varphi$ and $\theta(\lambda)$ are given by (13) and (29), respectively.

Therefore, using Hurwitz's theorem in the results of this theorem we obtain the same limit relation between the zeros of Hermite-Sobolev polynomials for case I and II and the zeros of the elementary trigonometric functions $\cos (x)$ and $\sin (x)$. That is,

Corollary 2.16 (Cor. 1 in [11] and Cor. 2 in [39]) With the notation of Theorem 2.14, we have

$$
\lim _{n \rightarrow \infty} 2 \sqrt{n} q_{2 n, j}^{(H k)}=(2 j-1) \frac{\pi}{2}, \quad \lim _{n \rightarrow \infty} 2 \sqrt{n} q_{2 n+1, j}^{(H k)}=j \pi, \quad j \in \mathbb{N}, \quad k=1,2 .
$$

Remark In [39] the Mehler-Heine type formulas as well as the zero asymptotics for the standard polynomials $W_{n}$ are deduced. In fact, the asymptotic behaviour of the smallest zeros of the Hermite polynomials $H_{n}$, the polynomials $W_{n}$ and the HermiteSobolev orthogonal polynomials (case I and II) is the same.

Now is the moment to analyze the question posed in pages 9 and 17: why the asymptotic behaviours for the cases II (Laguerre and Hermite) do not yield a constant as in the cases I? The answer is given taking into account the inner product:

$$
(f, g)=\int f(x) g(x) \mathrm{d} \mu_{0}(x)+\lambda \int f^{\prime}(x) g^{\prime}(x) \mathrm{d} \mu_{1}(x), \quad \lambda>0,
$$

where $\mu_{0}, \mu_{1}$ are positive Borel measures supported on the real axis. We can see that the derivative in the second integral introduce a multiplicative factor $n^{2}$ when we apply this inner product to monic polynomials of degree $n$. Therefore, in 'some sense' the measure $\mu_{1}$ plays the most important role and so in terms of relative asymptotics we should compare the Sobolev polynomials $Q_{n}$ with those associated with the second measure $\mu_{1}$. This was already observed in [2] and it was a motivation to introduce the Sobolev balanced extremal polynomials for measures with compact support. However, (16) and (30) allow us to give the outer strong asymptotics 
of the Laguerre-Sobolev and Hermite-Sobolev orthogonal polynomials in terms of the classical outer strong asymptotics of Laguerre and Hermite polynomials, respectively. Indeed, there are no differences between case I and case II in the relative asymptotics if we compare the Sobolev orthogonal polynomials with an adequate family of polynomials defined as:

$$
R_{n}(x)=\lim _{\lambda \rightarrow \infty} Q_{n}(x) .
$$

These polynomials have been known since the pioneering work [17] (see also for more details [28]) and they satisfy $R_{n}^{\prime}(x)=k_{n} P_{n-1}\left(x ; \mu_{1}\right)$ where $P_{n-1}\left(x ; \mu_{1}\right)$ are the polynomials orthogonal with respect to the measure $\mu_{1}$ and $k_{n}$ depends on the normalization. Now, we can simplify and list some results about relative asymptotics of Sobolev orthogonal polynomials:

\section{Proposition 2.17}

(a) Relative outer asymptotics.

$$
\lim _{n \rightarrow \infty} \frac{Q_{n}^{(L i)}(x)}{R_{n}^{(L i, \infty)}(x)}=\frac{1}{1-\ell}, \quad i=1,2
$$

uniformly on compact subsets of $\mathbb{C} \backslash \operatorname{supp}\left(\mu_{1}\right)$, where $R_{n}^{(L i, \infty)}(x)=$ $\lim _{\lambda \rightarrow \infty} Q_{n}^{(L i)}(x), i=1,2$, and $\ell$ is given by (17).

(b) Relative outer asymptotics.

$$
\lim _{n \rightarrow \infty} \frac{Q_{n}^{(H i)}(x)}{R_{n}^{(H i, \infty)}(x)}=\theta(\lambda), \quad i=1,2
$$

uniformly on compact subsets of $\mathbb{C} \backslash \mathbb{R}$, where $R_{n}^{(H i, \infty)}(x)=\lim _{\lambda \rightarrow \infty} Q_{n}^{(H i)}(x)$, $i=1,2, \varphi$ and $\theta(\lambda)$ are given by (13) and (29), respectively.

Proof For case I in Laguerre as well as in Hermite weights, the proof is straightforward because $R_{n}^{(L 1, \infty)}=L_{n}^{(\alpha-1)}(x)$ and $R_{n}^{(H 1, \infty)}(x)=H_{n}(x)$. On the other hand, for case II we have (see [5] and [37])

$$
R_{n}^{(L 2, \infty)}(x)=L_{n}^{(\alpha)}(x)-c_{n-1} L_{n-1}^{(\alpha)}(x)=L_{n}^{(\alpha-1)}(x)-\mathrm{d}_{n-1} L_{n-1}^{(\alpha)}(x),
$$

with $\left(R_{n}^{(L 2, \infty)}\right)^{\prime}(x)=-T_{n-1}(x)=-\left(L_{n-1}^{(\alpha+1)}(x)-c_{n-2} L_{n-2}^{(\alpha+1)}(x)\right)$, being $T_{n}$ the polynomials defined in page 170. Since

$$
\frac{Q_{n}^{(L 2)}(x)}{R_{n}^{(L 2, \infty)}(x)}=\frac{Q_{n}^{(L 2)}(x)}{L_{n}^{(\alpha-1)}(x)} \frac{\sqrt{n} L_{n}^{(\alpha-1)}(x)}{L_{n}^{(\alpha)}(x)} \frac{L_{n}^{(\alpha)}(x)}{T_{n}(x)} \frac{T_{n}(x)}{\sqrt{n} R_{n}^{(L 2, \infty)}(x)}
$$

it only remains to use (16), [37, Th.4.5, Lem.4.4] and the very well-known relative asymptotics for Laguerre polynomials of the different degrees which is deduced from Perron's Theorem (see [42, p.199]). Notice that in case I we have $\operatorname{supp}\left(\mu_{1}\right)=[0, \infty)$, but in case II associated with Laguerre weights, $\operatorname{supp}\left(\mu_{1}\right)=[0, \infty) \cup\{-\mathrm{a}\}$. This ensures that there is not a division by zero when we consider $\lim _{n \rightarrow \infty} L_{n}^{(\alpha)}(x) / T_{n}(x)$ (see [37, Remark 4.6]). On the other hand, the zeros of $R_{n}^{(L 2, \infty)}(x)$ accumulate on $[0, \infty)$ when $n \rightarrow \infty$.

(b) follows from Theorem 2 and Proposition 5 in [39] and taking into account that $R_{n}^{(H 2, \infty)}(x)$ has not complex zeros when $n \rightarrow \infty$ according to Theorem 6.3 in [13]. 


\section{The Freud Weights}

The first example of Sobolev inner product associated with measures of unbounded support out of the coherent or symmetric coherent pairs was given in [10] within the framework of the characterization of semiclassical measures. There the asymptotic properties of the Sobolev polynomials orthogonal with respect to the inner product,

$$
(p, q)=\int_{\mathbb{R}} p(x) q(x) e^{-x^{4}} \mathrm{~d} x+\lambda \int_{\mathbb{R}} p^{\prime}(x) q^{\prime}(x) e^{-x^{4}} \mathrm{~d} x, \quad \lambda>0,
$$

were deduced. Recently, in [18] a more general inner product was considered

$$
(f, g)=\int_{\mathbb{R}} f(x) g(x)(\psi(x) W(x))^{2} \mathrm{~d} x+\lambda \int_{\mathbb{R}} f^{\prime}(x) g^{\prime}(x) W^{2}(x) \mathrm{d} x
$$

where $\lambda>0, \psi \in L_{\infty}(\mathbb{R})$ is positive on a set of positive measure, and $W(x)=$ $\exp (-Q(x))$ is an exponential weight with some special assumptions over $Q(x)$. The techniques used in this paper are new due to the lack of an algebraic relation between these Sobolev orthogonal polynomials and standard polynomials associated with the weight function $W^{2}$.

We denote by $p_{n}=\gamma_{n} x^{n}+\ldots$ the orthonormal polynomials associated with $W^{2}$ and by $q_{n}=\kappa_{n} x^{n}+\ldots$ the Sobolev polynomials orthonormal with respect to the inner product (34). We also use the Mhaskar-Rakhmanov-Saff numbers (see, for example, [38]), $a_{n}, n \geq 1$, which are the positive roots of the equations

$$
n=\frac{2}{\pi} \int_{0}^{1} a_{n} t Q^{\prime}\left(a_{n} t\right) \frac{\mathrm{d} t}{\sqrt{1-t^{2}}}
$$

where $Q(x)$ is a convex and even function defined on $\mathbb{R}$. Notice that in our case $\lim _{n \rightarrow \infty} a_{n} / n=0$. Furthermore, we need the Szegó function (see [42]) associated with a measurable function $f:[-\pi, \pi] \rightarrow[0, \infty)$ satisfying the condition $\int_{-\pi}^{\pi} \log f(\theta) \mathrm{d} \theta>-\infty$, that is,

$$
D(f ; z)=\exp \left(\frac{1}{4 \pi} \int_{-\pi}^{\pi} \log f\left(e^{i t}\right) \frac{e^{i t}+z}{e^{i t}-z} \mathrm{~d} t\right), \quad|z|<1,
$$

with

$$
\left|D\left(f ; e^{i \theta}\right)\right|^{2}=f(\theta) \text { a.e. } \theta \in[-\pi, \pi] .
$$

The asymptotics of the polynomials $q_{n}$ and their derivatives are stated in the next:

Theorem 3.1 (Th. 1.1 in [18]) Let $Q: \mathbb{R} \rightarrow \mathbb{R}$ be an even and continuous function in $\mathbb{R}$. Assume that $Q^{\prime \prime}$ is continuous in $(0, \infty)$, and $Q^{\prime}>0$ in $(0, \infty)$. Assume, furthermore, that for some $\alpha, \beta>0$,

$$
\alpha \leq \frac{x Q^{\prime \prime}(x)}{Q^{\prime}(x)} \leq \beta, \quad x \in(0, \infty) .
$$


Then, using the previous notation, for $n \rightarrow \infty$,

$$
\left\|\left(q_{n}^{\prime}-\frac{1}{\sqrt{\lambda}} p_{n-1}\right) W\right\|_{L_{2}(\mathbb{R})}=O\left(\frac{a_{n}}{n}\right)=o(1)
$$

and

$$
\left\|\left(1+\left|Q^{\prime}\right|\right)\left(q_{n}-\frac{1}{\sqrt{\lambda}} \int_{0}^{x} p_{n-1}\right) W\right\|_{L_{2}(\mathbb{R})}=O\left(\sqrt{\frac{a_{n}}{n}}\right) .
$$

$$
\left\|\left(q_{n}^{\prime}-\frac{1}{\sqrt{\lambda}} p_{n-1}\right) W\right\|_{L_{\infty}(\mathbb{R})}=O\left(\sqrt{\frac{a_{n}}{n}}\right) .
$$

and

$$
\left\|\left(1+\left|Q^{\prime}\right|\right)\left(q_{n}-\frac{1}{\sqrt{\lambda}} \int_{0}^{x} p_{n-1}\right) W\right\|_{L_{\infty}(\mathbb{R})}=O\left(\sqrt{\frac{a_{n}}{n}}\right) .
$$

- Let

$$
W_{n}(\theta)=W\left(a_{n} \cos \theta\right), \quad \theta \in[-\pi, \pi] .
$$

Uniformly in closed subsets of $\mathbb{C} \backslash[-1,1]$,

$$
\left|\frac{\left(q_{n}^{\prime}-\frac{1}{\sqrt{\lambda}} p_{n-1}\right)\left(a_{n} z\right)}{\varphi(z)^{n-1} D^{-2}\left(W_{n} ; 1 / \varphi(z)\right)}\right|=O\left(\frac{\sqrt{a_{n}}}{n}\right) .
$$

Uniformly in closed subsets of $\mathbb{C} \backslash[-1,1]$,

$$
\left|\frac{q_{n}\left(a_{n} z\right)-\frac{1}{\sqrt{\lambda}} \int_{0}^{a_{n} z} p_{n-1}}{\varphi(z)^{n} D^{-2}\left(W_{n} ; 1 / \varphi(z)\right)}\right|=O\left(\frac{a_{n}^{3 / 2}}{n^{2}}\right),
$$

where $\varphi$ is given by (13).

Outlines of the proof To establish these results we need to introduce some concepts. Let $f$ be an absolutely continuous function, then

$$
E_{n}[f ; W]=\inf \left\{\|(f-P) W\|_{L_{2}(\mathbb{R})}: \operatorname{deg}(P) \leq n\right\}
$$

is the weighted $L_{2}$ error of approximation. Let

$$
I[R](x)=W(x)^{-2} \int_{-\infty}^{x} R(t) W^{2}(t) \psi^{2}(t) \mathrm{d} t, \quad x \in \mathbb{R},
$$

be the linear operator defined on suitably restricted classes of functions $R$.

We begin giving the main ideas of the proof of (36).

- In the framework of more general weights (see [18, proof of Th. 1.3]) an upper bound for the ratio $\gamma_{n-1} / \gamma_{n}$ depending of $n$ is proved, that is,

$$
\frac{\gamma_{n-1}}{\gamma_{n}} \leq 4 a_{n}
$$


- Using the Jackson inequality for exponential weights (see, for example, [14])

$$
E_{n}[f ; W] \leq C \frac{a_{n}}{n}\left\|f^{\prime} W\right\|_{L_{2}(\mathbb{R})},
$$

the statement of Theorem 1.5 in [18] asserts that

$$
\left|\left(\frac{\gamma_{n-1}}{n \kappa_{n}}\right)^{2}-\lambda\right| \leq\left(\|\psi\|_{L_{\infty}(\mathbb{R})} \frac{\gamma_{n-1}}{n \gamma_{n}}\right)^{2}+C_{1}\left(\frac{a_{n-2}}{n-2}\right)^{2} .
$$

Now, using (42) and the properties of $\psi$ we obtain

$$
\left|\left(\frac{\gamma_{n-1}}{n \kappa_{n}}\right)^{2}-\lambda\right|=O\left(\frac{a_{n}}{n}\right)^{2} .
$$

- From the Sobolev orthogonality of the polynomials $q_{n}$ we get

$$
\int_{\mathbb{R}}\left(q_{n}^{\prime}-n \frac{\kappa_{n}}{\gamma_{n-1}} p_{n-1}\right)^{2} W^{2} \leq \frac{1}{\lambda}-\left(n \frac{\kappa_{n}}{\gamma_{n-1}}\right)^{2}
$$

- Finally, taking into account

$$
\begin{aligned}
\int_{\mathbb{R}}\left(q_{n}^{\prime}-\frac{1}{\sqrt{\lambda}} p_{n-1}\right)^{2} W^{2}= & \int_{\mathbb{R}}\left(q_{n}^{\prime}-n \frac{\kappa_{n}}{\gamma_{n-1}} p_{n-1}\right)^{2} W^{2} \\
& +\int_{\mathbb{R}}\left(n \frac{\kappa_{n}}{\gamma_{n-1}}-\frac{1}{\sqrt{\lambda}}\right)^{2} p_{n-1}^{2} W^{2},
\end{aligned}
$$

it only remains to use (43) and (44) in order to obtain (36).

Equation (37) follows from four results: (36), [18, Lem.3.4.(c)], that is,

$$
\left\|\left(1+\left|Q^{\prime}\right|\right)\left(q_{n}-q_{n}(0)-\frac{1}{\sqrt{\lambda}} \int_{0}^{x} p_{n-1}\right) W\right\|_{L_{2}(\mathbb{R})}=O\left(\frac{a_{n}}{n}\right),
$$

the bound

$$
\left|q_{n}(0)\right| \leq C \sqrt{\frac{a_{n}}{n}}
$$

given in [18, Lem.5.2], and the fact that $\left\|\left(1+\left|Q^{\prime}\right|\right) W\right\|_{L_{2}(\mathbb{R})}$ is finite.

To prove (38) we only need (36) and the Nikolskii inequality $\|P W\|_{L_{\infty}(\mathbb{R}) \leq}$ $C\left(\frac{n}{a_{n}}\right)^{1 / 2}\|P W\|_{L_{2}(\mathbb{R})}$. The proof of (39) follows the proof of (37) but now we use (38) and

$$
\left\|\left(1+\left|Q^{\prime}\right|\right)\left(q_{n}-q_{n}(0)-\frac{1}{\sqrt{\lambda}} \int_{0}^{x} p_{n-1}\right) W\right\|_{L_{\infty}(\mathbb{R})}=O\left(\sqrt{\frac{a_{n}}{n}}\right) .
$$

Finally, (40) follows from (36) and applying Lemma 14.6 in [21, p.395] with $p=$ $2, m=n-1$, and $w=W_{n}$. To establish (41) we need this Lemma 14.6, (45) and (46), and some other technical results. 
From this theorem and very well-known results for orthogonal polynomials associated with exponential weights, see for example [21], we deduce:

Corollary 3.2 (Cor. 1.2 in [18])

(a) When $n$ tends to infinity

$$
\kappa_{n}=\frac{1}{n} \frac{1}{\sqrt{2 \pi \lambda}}\left(\frac{a_{n}}{2}\right)^{-n+\frac{1}{2}} \exp \left(\frac{2}{\pi} \int_{0}^{1} \frac{Q\left(a_{n} s\right)}{\sqrt{1-s^{2}}} \mathrm{~d} s\right)(1+o(1)) .
$$

(b) For $n \rightarrow \infty$,

$$
\begin{aligned}
& \int_{-1}^{1} \mid \sqrt{\lambda a_{n}} q_{n}^{\prime}\left(a_{n} x\right) W\left(a_{n} x\right)-\frac{\sqrt{2 / \pi}}{\left(1-x^{2}\right)^{1 / 4}} \\
& \quad \times\left.\cos \left[\left(n-\frac{1}{2}\right) \arccos x+2 \Gamma\left(W_{n} ; \arccos x\right)-\frac{\pi}{4}\right]\right|^{2} \mathrm{~d} x=o(1) .
\end{aligned}
$$

(c) Uniformly for $z$ in closed subsets of $\mathbb{C} \backslash[-1,1]$, we have, as $n \rightarrow \infty$,

$$
\begin{gathered}
\sqrt{\lambda a_{n}} q_{n}^{\prime}\left(a_{n} z\right) /\left\{\varphi(z)^{n-1} D^{-2}\left(W_{n} ; \frac{1}{\varphi(z)}\right)\left(1-\varphi(z)^{-2}\right)^{-1 / 2}\right\} \\
=\frac{1}{\sqrt{\pi}}(1+o(1)) .
\end{gathered}
$$

(d) There exists $\eta>0$ such that as $n \rightarrow \infty$, we have, uniformly for $|x| \leq 1-n^{-\eta}$, $x=\cos \theta$,

$$
\begin{aligned}
\sqrt{\lambda a_{n}} q_{n}^{\prime}\left(a_{n} x\right) W\left(a_{n} x\right)\left(1-x^{2}\right)^{1 / 4}= & \sqrt{\frac{2}{\pi}} \cos \left(\left(n-\frac{1}{2}\right) \theta+2 \Gamma\left(W_{n} ; \theta\right)-\frac{\pi}{4}\right) \\
& +O\left(n^{-\eta}\right)
\end{aligned}
$$

\section{Remarks}

- Theorem 3.1 can be formulated for more general weights, see Theorem 1.3 in [18], even for weights such that the asymptotics for the corresponding orthogonal polynomials is unknown, and therefore the previous Corollary cannot be established.

- The main key to obtain (36) and, therefore some of the other asymptotic properties of the polynomials $q_{n}$, is [18, formula (34) in Th.1.5]. To obtain this formula we need a Jackson inequality for exponential weights and bounds of the sequence $\left(\gamma_{n-1} /\left(n \kappa_{n}\right)\right)^{2}$ given in Theorem 1.4 of [18], i.e.,

$$
\lambda+\left(\frac{\gamma_{n-1}}{n \gamma_{n}^{*}}\right)^{2} \leq\left(\frac{\gamma_{n-1}}{n \kappa_{n}}\right)^{2} \leq \lambda+\left(\frac{\gamma_{n-1}}{n \gamma_{n}^{*}}\right)^{2}+\sup E_{n-2}^{2}[I[R] ; W],
$$

where $\gamma_{n}^{*}$ is the leading coefficient of the orthonormal polynomials $\pi_{n}$ for the weight function $(\psi W)^{2}$, and where the sup is taken over all polynomials $R$ of degree $\leq n-1$ satisfying both

$$
\|R W \psi\|_{L_{2}(\mathbb{R})}=1 \quad \text { and } \int_{\mathbb{R}} R(W \psi)^{2}=0 .
$$


Theorem 1.4 in [18] is essential to establish Theorem 1.1 as well as Theorem 1.3 for more general weights in [18]. So, it is important to point out that the inequalities given by (47) for the leading coefficients are equivalent to the ones for the square of the norms used to establish the asymptotics of the Sobolev orthogonal polynomials in the framework of the coherence (see, for example, [2, p.52],[4, proof of Lemma 2.6],[29, Prop.3] or [33, Th.2]). Furthermore, these inequalities for the square of the norms can be obtained when we have a finite algebraic relation between Sobolev polynomials and the standard ones (one example is given in [10]).

On the other hand, the lower bound for $\left(\gamma_{n-1} /\left(n \kappa_{n}\right)\right)^{2}$ given in (47) is a 'universal' property of the Sobolev orthogonality, that is, if we consider the monic polynomials $\Pi_{n}=\pi_{n} / \gamma_{n}^{*}, P_{n}=p_{n} / \gamma_{n}$, and $Q_{n}=q_{n} / \kappa_{n}$ we have,

$$
\begin{aligned}
\left(Q_{n}, Q_{n}\right)= & \int Q_{n}^{2}(x)(\psi W)^{2}(x) \mathrm{d} x+\lambda \int\left(Q_{n}^{\prime}\right)^{2}(x) W^{2}(x) \mathrm{d} x \\
& \geq \int \Pi_{n}^{2}(x)(\psi W)^{2}(x) \mathrm{d} x+\lambda n^{2} \int P_{n-1}^{2}(x) W^{2}(x) \mathrm{d} x .
\end{aligned}
$$

where the extremal property of the square of the norms of $\Pi_{n}$ and $P_{n}$ have been used. Now, this relation can be expressed in terms of the leading coefficients as

$$
\frac{1}{\kappa_{n}^{2}} \geq\left(\frac{1}{\gamma_{n}^{*}}\right)^{2}+\lambda\left(\frac{n}{\gamma_{n-1}}\right)^{2}
$$

from where the inequality on the left of (47) follows. However, until the work [18], an upper bound for $\left(\gamma_{n-1} /\left(n \kappa_{n}\right)\right)^{2}$ had been obtained only assuming the existence of a finite algebraic relation between Sobolev and standard polynomials. Therefore, the upper bound given in (47) is an important advance in the study of Sobolev orthogonal polynomials.

There are not a lot of results about the zeros of these Sobolev polynomials for Freud weights. For the polynomials orthogonal with respect to the particular inner product (33) it has been proved that the zeros, $q_{n, k}^{\lambda}$, of the corresponding Sobolev orthogonal polynomials are real, simple, interlace with those of the standard polynomials orthogonal for the weight function $e^{-x^{4}}$ on $\mathbb{R}$, and for $n$ fixed each positive zero $q_{n, k}^{\lambda}, k=1, \ldots,[n / 2]$, is an increasing function of $\lambda$ (see [40]). Are the zeros of Sobolev polynomials for exponential weights like those considered in [18] real and simple? This question remains open.

On the other hand, in a very recent work [23] the conditions on the weights in [18] have been relaxed aiming for weak asymptotic instead of strong asymptotic results. In fact, for $p \in[1, \infty)$ the authors consider a family of weights $\left\{w_{i}\right\}_{i=0}^{m}$ and the Sobolev $p$-norm

$$
\|q\|_{S}=\left(\sum_{k=0}^{m} \int_{\mathbb{R}}\left|q^{(k)}(x) w_{k}(x)\right|^{p} \mathrm{~d} x\right)^{1 / p}=\left(\sum_{k=0}^{m}\left\|q^{(k)} w_{k}\right\|_{L^{p}(\mathbb{R})}^{p}\right)^{1 / p}, q \in \mathbb{P},
$$

where $\mathbb{P}$ is the linear space of polynomials with real coefficients. Then, it is said that $Q_{n}$ is a $n$-th extremal monic polynomial with respect to (48) if

$$
\left\|Q_{n}\right\|_{S}=\min \left\{\|q\|_{S}: q(x)=x^{n}+\ldots\right\} .
$$


The existence of such a type of polynomials is easy to prove when $n \in \mathbb{Z}_{+}$. Next, we use the following definition:

Definition 3.3 Let $w$ be a positive continuous function on $\mathbb{R}$.

- $\quad w \in W(\alpha, \tau)$ with $\alpha, \tau>0$, if

$$
\lim _{|x| \rightarrow \infty} \frac{-\log w(x)}{\tau|x|^{\alpha}}=1
$$

- $\quad w \in W(\alpha)$ with $\alpha>0$, if

$$
\lim _{|x| \rightarrow \infty} \frac{\log \log \frac{1}{w(x)}}{\log |x|}=\alpha .
$$

Notice that $W(\alpha, \tau) \subset W(\alpha)$, for all $\tau>0$. In particular, the Freud-type weights $e^{-\tau|x|^{\alpha}} \in W(\alpha, \tau)$.

Let $\bar{k} \in\{0,1, \ldots, m\}$ be the smallest index such that either

$$
\alpha_{\bar{k}}<\min _{\substack{0 \leq k \leq m \\ k \neq \bar{k}}} \alpha_{k}, \quad \text { or } \quad \alpha_{\bar{k}}=\min _{\substack{0 \leq k \leq m \\ k \neq \bar{k}}} \alpha_{k} \quad \text { and } \quad \tau_{\bar{k}}=\min \left\{\tau_{k}: \alpha_{k}=\alpha_{\bar{k}}\right\}
$$

and let $\tilde{k} \in\{0,1, \ldots, m\}$ be the first index such that

$$
\alpha_{\tilde{k}}=\min \left\{\alpha_{k}: 0 \leq k \leq m\right\}
$$

We also use the notation $\bar{\alpha}=\alpha_{\bar{k}}, \bar{\tau}=\tau_{\bar{k}}$, and $\widetilde{\alpha}=\alpha_{\tilde{k}}$. In [23], the $n$-th root and logarithmic asymptotics of $Q_{n}$ are established:

Theorem 3.4 (Th. 1.1 and 1.2 in [23])

(a) Let $Q_{n}$ be the $n$-th Sobolev monic extremal polynomial relative to the norm (48) where $w_{k} \in W\left(\alpha_{k}, \tau_{k}\right), 0 \leq k \leq m$. Then

$$
\lim _{n \rightarrow \infty} n^{-1 / \bar{\alpha}}|| Q_{n} \|_{S}^{1 / n}=\frac{1}{2}\left(\frac{\gamma_{\bar{\alpha}}}{\bar{\tau} e}\right)^{1 / \bar{\alpha}},
$$

and, for all $j \geq \bar{k}$

$$
\lim _{n \rightarrow \infty} n^{-1 / \bar{\alpha}}|| Q_{n}^{(j)} e^{-\bar{\tau}|x|^{\bar{\alpha}}} \|_{L_{\infty(\mathbb{R})}^{1 / n}}=\frac{1}{2}\left(\frac{\gamma_{\bar{\alpha}}}{\bar{\tau} e}\right)^{1 / \bar{\alpha}}
$$

where

$$
\gamma_{\bar{\alpha}}=\frac{\Gamma(\bar{\alpha} / 2) \Gamma(1 / 2)}{2 \Gamma((1+\bar{\alpha}) / 2)} .
$$

(b) Let $Q_{n}$ be the $n$-th Sobolev monic extremal polynomial relative to the norm (48) where $w_{k} \in W\left(\alpha_{k}\right), 0 \leq k \leq m$. Then

$$
\lim _{n \rightarrow \infty}\left\|Q_{n}\right\|_{S}^{1 /(n \log n)}=e^{1 / \widetilde{\alpha}}
$$




\section{Remarks}

- The asymptotic contracted limit distribution of the zeros of $Q_{n}^{(j)}$ and the weak limit of the corresponding contracted extremal polynomials is obtained in [23, Cor.3.1 and 3.2] as a consequence of (49).

- Some of the techniques used to prove these results are similar to those used in [24] and [25] for the bounded case.

\section{Some Open Problems and New Directions}

In the previous sections some unsolved questions and conjectures have been posed in their natural context. Next, we wish to give some guidelines which we consider as new directions in the study of the analytical properties of Sobolev orthogonal polynomials associated with measures with unbounded support.

1. Geronimo et al. [18] permits the study of analytic properties of the polynomials orthogonal with respect to the inner product

$$
(p, q)=\int p(x) q(x) \mathrm{d} \mu_{0}+\lambda \int_{0}^{\infty} p^{\prime}(x) q^{\prime}(x) x^{\alpha} e^{-x} \mathrm{~d} x, \quad \alpha>-1,
$$

where the measure $\mu_{0}$ is in some sense 'admissible', for example, $\mathrm{d} \mu_{0}(x)=$ $\psi(x) x^{\alpha} e^{-x} \mathrm{~d} x$ where $\psi$ satisfies suitable conditions. Under these assumptions it is possible to establish an analog of Theorem 1.4 in [18].

2. In this paper we have dealt with a Sobolev inner product involving the derivative operator. However, another possibility is to consider the inner product

$$
(p, q)=\int_{\mathbb{R}} p(x) q(x) \mathrm{d} \mu_{0}(x)+\lambda \int_{\mathbb{R}}(\Delta p)(x)(\Delta q)(x) \mathrm{d} \mu_{1}(x),
$$

where $\mu_{0}, \mu_{1}$ are measures supported on a countable set and $(\Delta p)(c)=p(c+$ $1)-p(c)$ denotes the forward difference operator. The goal of this type of inner product is to show that a family of continuous Sobolev orthogonal polynomials can be seen as a limit of the $\Delta$-Sobolev polynomials orthogonal with respect to the inner product (50). Notice that in [9] an inner product like (50) with $\mu_{1}$ as the measure corresponding to the Meixner weight function was considered in the framework of $\Delta$-coherence. More precisely, according to the classification of $\Delta$-coherent pairs (see [8]) if $\mu_{1}$ is a classical discrete measure (in our case, Meixner) then $\mu_{0}$ is a polynomial modification of degree one of the measure $\mu_{1}$.

We denote by $M_{n}^{(\gamma, \mu)}(x)$ the monic Meixner polynomials orthogonal with respect to

$$
(p, q)=\sum_{x=0}^{\infty} p(x) q(x) \frac{\mu^{x} \Gamma(x+\gamma)(1-\mu)^{\gamma}}{\Gamma(\gamma) \Gamma(x+1)}, \quad \gamma>0, \quad 0<\mu<1,
$$

and by $Q_{n}(x ; \gamma, \mu ; \lambda, K, a)$ the $\Delta$-Meixner-Sobolev polynomial orthogonal with respect to (50) where $\left(\mu_{0}, \mu_{1}\right)$ is a $\Delta$-coherent pair of measures of case I (Meixner), that is, $\mu_{1}$ is the Meixner measure. Notice that the parameters $a, K$ appear in the expression of the measure $\mu_{0}$ (see [9, Prop. 2.10]). In [9] the outer asymptotics of these polynomials $Q_{n}(x ; \gamma, \mu ; \lambda, K, a)$ was studied. 
Furthermore, some algebraic and analytic results for coherent pairs associated with Laguerre weights were recovered in Section 4 of that work. In particular, denoting by $Q_{n}(x ; \alpha ; \lambda, M, a):=(-1)^{n} n ! Q_{n}^{(L 1)}(x)$ being $Q_{n}^{(L 1)}(x)$ the LaguerreSobolev orthogonal polynomials defined in Theorem 2.5, then (see [9, Prop. 4.4])

$$
\lim _{\mu \uparrow 1}(1-\mu)^{n} Q_{n}\left(\frac{x}{1-\mu} ; \alpha+1, \mu ; \frac{\lambda}{(1-\mu)^{2}}, M, \frac{a}{1-\mu}\right)=Q_{n}(x ; \alpha ; \lambda, M, a) .
$$

Equation (51) is a limit relation between $\Delta$-Meixner-Sobolev orthogonal polynomials and the Laguerre-Sobolev ones which is similar to the one existing between Meixner and Laguerre polynomials in the Askey scheme (see, for example, [7]). A natural consequence is the possibility of establishing a SobolevAskey scheme.

3. As we have commented previously, the second measure $\mu_{1}$ plays the main role in the Sobolev orthogonality. In order to equalize the role of both measures, the balanced Sobolev orthogonal polynomials were introduced in [2] where both measures have compact support. It seems natural that we can use this approach for the study of Sobolev orthogonal polynomials in the unbounded case, although this presents some complications. We are working in this direction, and we have a new result where we show how to balance in the unbounded case (see [3]).

4. We consider the Sobolev-type inner product:

$$
(p, q)=\int_{I} p(x) q(x) \mathrm{d} \mu+\sum_{i=0}^{N} M_{i} p^{(i)}(c) q^{(i)}(c), \quad M_{i} \geq 0, c \in \mathbb{R},
$$

where $\mu$ is a positive measure with unbounded support $I \subseteq \mathbb{R}$. Algebraic results for the orthogonal polynomials can be deduced regardless whether the measure $\mu$ is unbounded or not. The study of the asymptotics for the polynomials orthogonal with respect to (52) has been done especially when $\mu$ is bounded (see the survey [32]), and recently in [6] when $\mu$ is the measure associated with the Laguerre weight, $N=1$, and $c=0$. Therefore, the study of the asymptotics and the zero asymptotics of the polynomials orthogonal with respect to (52) when the measure $\mu$ is more general, for example associated with Freud weights, remains open.

5. Finally, a very interesting problem is to consider the inner product:

$$
(p, q)=\int_{I_{0}} p(x) q(x) \mathrm{d} \mu_{0}(x)+\lambda \int_{I_{1}} p^{\prime}(x) q^{\prime}(x) \mathrm{d} \mu_{1}(x),
$$

where $\mu_{i}$ are positive measures supported on $I_{i} \subseteq \mathbb{R}, i=1,2$, respectively, with non-zero absolutely continuous parts, and $I_{0} \cap I_{1}=\emptyset$ or, at least, $I_{0} \supset I_{1}$. The main work in this direction is [15] where the authors consider measures with compact support under some general assumptions and they study the asymptotic distribution of the zeros and critical points of the polynomials orthogonal with respect to (53), and they formulated some conjectures. As far as we know, there are no more works in this direction, and therefore this is an open problem for both the unbounded and the bounded cases. 
Acknowledgements The authors thank the referee by the careful revision of the manuscript and the suggestions for improve the presentation.

\section{References}

1. Alfaro, M., López, G., Rezola, M.L.: Some properties of zeros of Sobolev-type orthogonal polynomials. J. Comput. Appl. Math. 69, 171-179 (1996)

2. Alfaro, M., Martínez-Finkelshtein, A., Rezola, M.L.: Asymptotics properties of balanced extremal Sobolev polynomials: coherent case. J. Approx. Theory 100, 44-59 (1999)

3. Alfaro, M., Moreno-Balcázar, J.J., Peña, A., Rezola, M.L.: Sobolev orthogonal polynomials: how to balance and asymptotics (submitted for publication).

4. Alfaro, M., Moreno-Balcázar, J.J., Pérez, T.E., Piñar, M.A., Rezola, M.L.: Asymptotics of Sobolev orthogonal polynomials for Hermite coherent pairs. J. Comput. Appl. Math. 133, 141$150(2001)$

5. Alfaro, M., Moreno-Balcázar, J.J., Rezola, M.L.: Laguerre-Sobolev orthogonal polynomials: asymptotics for coherent pairs of type II. J. Approx. Theory 122, 79-96 (2003)

6. Alvarez-Nodarse, R., Moreno-Balcázar, J.J.: Asymptotic properties of generalized Laguerre orthogonal polynomials. Indag. Math. (N.S.) 15, 151-165 (2004)

7. Andrews, G.E., Askey, R.: Classical orthogonal polynomials. In: Brezinski, C. et al. (eds.) Polynômes Orthogonaux et Applications, Lecture Notes in Mathematics, vol. 1171, pp. 36-62. Springer, Berlin Heidelberg New York (1985)

8. Area, I., Godoy, E., Marcellán, F.: Classification of all $\Delta$-coherent pairs. Integral Transform. Spec. Funct. 9(1), 1-18 (2000)

9. Area, I., Godoy, E., Marcellán, F., Moreno-Balcázar, J.J.: $\Delta$-Sobolev orthogonal polynomials of Meixner type: asymptotics and limit relation. J. Comput. Appl. Math. 178, 21-36 (2005)

10. Cachafeiro, A., Marcellán, F., Moreno-Balcázar, J.J.: On asymptotic properties of FreudSobolev orthogonal polynomials. J. Approx. Theory 125, 26-41 (2003)

11. Castaño-García, L., Moreno-Balcázar, J.J.: A Mehler-Heine type formula for Hermite-Sobolev orthogonal polynomials. J. Comput. Appl. Math. 150, 25-35 (2003)

12. de Bruin, M.G., Groenevelt, W.G.M., Marcellán, F., Meijer, H.G., Moreno-Balcázar, J.J.: Asymptotics and zeros of symmetrically coherent pairs of Hermite type (submitted for publication).

13. de Bruin, M.G., Groenevelt, W.G.M., Meijer, H.G.: Zeros of Sobolev orthogonal polynomials of Hermite type. Appl. Math. Comput. 132, 135-166 (2002)

14. Ditzian, Z., Lubinsky, D.S.: Jackson and smoothness theorems for Freud weights in $L_{p}(0<p \leq$ $\infty)$. Constr. Approx. 13, 99-152 (1997)

15. Gautschi, W., Kuijlaars, A.B.J.: Zeros and critical points of Sobolev orthogonal polynomials. J. Approx. Theory 91, 117-137 (1997)

16. Iserles, A., Koch, P.E., Nørsett, S.P., Sanz-Serna, J.M.: Orthogonality and approximation in a Sobolev space. In: Mason, J.C., Cox, M.G. (eds.) Algorithms for Approximation, pp. 117-124. Chapman \& Hall, London, UK (1990)

17. Iserles, A., Koch, P.E., Nørsett, S.P., Sanz-Serna, J.M.: On polynomials orthogonal with respect to certain Sobolev inner products. J. Approx. Theory 65, 151-175 (1991)

18. Geronimo, J.S., Lubinsky, D.S., Marcellán, F.: Asymptotics for Sobolev orthogonal polynomials for exponential weights. Constr. Approx. 22, 309-346 (2005)

19. Geronimo, J.S., Van Assche, W.: Relative asymptotics for orthogonal polynomials with unbounded recurrence coefficients. J. Approx. Theory 62, 47-69 (1990)

20. Koekoek, R., Meijer, H.G.: A generalization of Laguerre polynomials. SIAM J. Math. Anal. 24(3), 768-782 (1993)

21. Levin, E., Lubinsky, D.S.: Orthogonal Polynomials for Exponential Weights. Springer, Berlin Heidelberg New York (2001)

22. Lewis, D.C.: Polynomial least square approximations. Amer. J. Math. 69, 273-278 (1947)

23. López-Lagomasino, G., Marcellán, F., Pijeira, H.: Logarithmic asymptotic of contracted Sobolev extremal polynomials on the real line. J. Approx. Theory (in press)

24. López-Lagomasino, G., Pijeira, H.: Zero location and n-th root asymptotics de Sobolev orthogonal polynomials. J. Approx. Theory 99, 30-43 (1999)

25. López-Lagomasino, G., Pijeira, H., Pérez, I.: Sobolev orthogonal polynomials in the complex plane. J. Comput. Appl. Math. 127, 219-230 (2001) 
26. Marcellán, F., Alfaro, M., Rezola, M.L.: Orthogonal polynomials on Sobolev spaces: old and new directions. J. Comput. Appl. Math. 48, 113-131 (1993)

27. Marcellán, F., Meijer, H.G., Pérez, T.E., Piñar, M.A.: An asymptotic result for Laguerre-Sobolev orthogonal polynomials. J. Comput. Appl. Math. 87, 87-94 (1997)

28. Marcellán, F., Martínez-Finkelshtein, A., Moreno-Balcázar, J.J.: $k$-coherence of measures with non-classical weights. In: Español, L., Varona, J.L.(eds.) Margarita Mathematica en memoria de José Javier (Chicho) Guadalupe Hernández, pp. 77-83. Servicio de Publicaciones Universitario de La Rioja, Spain (2001)

29. Marcellán, F., Moreno-Balcázar, J.J.: Strong and Plancherel-Rotach asymptotics of nondiagonal Laguerre-Sobolev orthogonal polynomials. J. Approx. Theory 110, 54-73 (2001)

30. Marcellán, F., Ronveaux, A.: A bibliography of Sobolev orthogonal polynomials. Internal Report. Universidad Carlos III de Madrid, Spain (July 2005)

31. Martínez-Finkelshtein, A.: Asymptotic properties of Sobolev orthogonal polynomials. J. Comput. Appl. Math. 99, 491-510 (1998)

32. Martínez-Finkelshtein, A.: Analytic aspects of Sobolev orthogonal polynomials revisited. J. Comput. Appl. Math. 127, 255-266 (2001)

33. Martínez-Finkelshtein, A., Moreno-Balcázar, J.J., Pérez, T.E., Piñar, M.A.: Asymptotics of Sobolev orthogonal polynomials for coherent pairs of measures. J. Approx. Theory 92, 280-293 (1998)

34. Meijer, H.G.: A short history of orthogonal polynomials in a Sobolev space. I. The non-discrete case. Nieuw Arch. Wisk. 14, 93-112 (1996)

35. Meijer, H.G.: Determination of all coherent pairs. J. Approx. Theory 89, 321-343 (1997)

36. Meijer, H.G., de Bruin, M.G.: Zeros of Sobolev orthogonal polynomials following from coherent pairs. J. Comput. Appl. Math. 139, 253-274 (2002)

37. Meijer, H.G., Pérez, T.E., Piñar, M.A.: Asymptotics of Sobolev orthogonal polynomials for coherent pairs of Laguerre type. J. Math. Anal. Appl. 245, 528-546 (2000)

38. Mhaskar, H.N., Saff, E.B.: Extremal problems for polynomials with exponential weights. Trans. Amer. Math. Soc. 285, 204-234 (1984)

39. Moreno-Balcázar, J.J.: Smallest zeros of some types of orthogonal polynomials: asymptotics. J. Comput. Appl. Math. 179, 289-301 (2005)

40. Moreno-Balcázar, J.J.: A note on the zeros of Freud-Sobolev orthogonal polynomials. J. Comput. Appl. Math. (in press)

41. Pan, K.: On Sobolev orthogonal polynomials with coherent pairs: the Laguerre case, type I. J. Math. Anal. Appl. 223, 319-334 (1998)

42. Szegő, G.: Orthogonal polynomials. Amer. Math. Soc. Colloq. Publ. 23, 4th edn. Amer. Math. Soc., Providence, Rhode Island (1975) 